\title{
CREB SUMOylation by the E3 Ligase PIAS1 Enhances Spatial Memory
}

\author{
Yan-Chu Chen, ${ }^{1 \star}$ Wei-Lun Hsu, ${ }^{2 \star}$ Yun-Li Ma, ${ }^{2}$ Derek J.C. Tai, ${ }^{2}$ and Eminy H.Y. Lee ${ }^{1,2}$ \\ ${ }^{1}$ Graduate Institute of Life Sciences, National Defense Medical Center, and ${ }^{2}$ Institute of Biomedical Sciences, Academia Sinica, Taipei 115, Taiwan, Republic \\ of China
}

cAMP-responsive element binding protein (CREB) phosphorylation and signaling plays an important role in long-term memory formation, but other posttranslational modifications of CREB are less known. Here, we found that CREB $1 \Delta$, the short isoform of CREB, could be sumoylated by the small ubiquitin-like modifier (SUMO) E3 ligase protein inhibitor of activated STAT1 (PIAS1) at Lys271 and Lys290 and PIAS1 SUMOylation of CREB1 $\Delta$ increased the expression level of CREB1 $\Delta$. CREB1 $\Delta$ could also be sumoylated by other PIAS family proteins, but not by the E3 ligases RanBP2 and Pc2 or by the E2 ligase Ubc9. Furthermore, water maze training increased the level of endogenous CREB SUMOylation in rat CA1 neurons determined by in vitro SUMOylation assay, but this effect was not observed in other brain areas. Moreover, transduction of Lenti-CREBWT to rat CA1 area facilitated, whereas transduction of Lenti-CREB double sumomutant (CREBK271RK290R) impaired, spatial learning and memory performance. Transduction of Lenti-CREBWT-SUM01 fusion vector to rat CA1 area showed a more significant effect in enhancing spatial learning and memory and CREB SUMOylation. Lenti-CREBWT transduction increased, whereas Lenti-CREBK271RK290R transduction decreased, CREB DNA binding to the brain-derived neurotrophic factor $(b d n f)$ promoter and decreased $b d n f$ mRNA expression. Knock-down of PIAS1 expression in CA1 area by PIAS1 siRNA transfection impaired spatial learning and memory and decreased endogenous CREB SUMOylation. In addition, CREB SUMOylation was CREB phosphorylation dependent and lasted longer. Therefore, CREB phosphorylation may be responsible for signal transduction during the early phase of long-term memory formation, whereas CREB SUMOylation sustains long-term memory.

Key words: CREB; phosphorylation; PIAS1; spatial learning and memory; SUMOylation

\section{Introduction}

Protein inhibitor of activated STAT1 (PIAS1) was initially identified as an inhibitor of STAT1 that blocks the DNA-binding activity of STAT1 and inhibits STAT1-mediated gene transcription in response to cytokine stimulation (Liu et al., 1998; Liao et al., 2000). PIAS1 also inhibits interferon-inducible gene expression and plays an important role in the innate immune response through negative regulation of STAT1 (Liu et al., 2004). Further studies indicated that PIAS1 also regulates the activity of other transcription factors to regulate immune response, such as NF- $\kappa \mathrm{B}$ and Smad4 (Shuai and Liu, 2003). However, other physiological functions that PIAS1 also participates in are less well known.

Recently, we demonstrated that PIAS1 facilitates spatial memory formation in rats. The expression level of PIAS1 is increased in the hippocampal CA1 area after water maze training. Further-

\footnotetext{
Received Oct. 8, 2013; revised May 25, 2014; accepted June 2, 2014.

Author contributions: Y.-C.C., W.-L.H., and E.H.Y.L. designed research; Y.-C.C., W.-L.H., Y.-L.M., and D.J.C.T. performed research; Y.-C.C., W.-L.H., Y.-L.M., and D.J.C.T. analyzed data; Y.-C.C., W.-L.H., and E.H.Y.L. wrote the paper.

This work was supported by the National Science Council of Taiwan (Grant NSC 101-2321-B-001-045). We thank the staff of the sequencing core facility of the Institute of Biomedical Sciences, Academia Sinica.

The authors declare no competing financial interests.

*Y.-C.C. and W.-L.H. contributed equally to this work.

Correspondence should be addressed to Dr. Eminy H.Y. Lee, Ph.D., Institute of Biomedical Sciences, Academia Sinica, Taipei 115, Taiwan. E-mail: eminy@gate.sinica.edu.tw.

DOI:10.1523/JNEUROSCI.4302-13.2014

Copyright $\odot 2014$ the authors $\quad 0270-6474 / 14 / 349574-16 \$ 15.00 / 0$
}

more, overexpression of PIAS1 in the CA1 neurons facilitates, whereas knock-down of PIAS1 expression by PIAS1 siRNA transfection impairs, spatial learning and memory performance in rats (Tai et al., 2011). Moreover, PIAS1 was found to facilitate spatial learning and memory through enhanced SUMOylation of STAT1 and decreased phosphorylation of STAT1 (Tai et al., 2011), but other mechanisms underlying PIAS1-mediated memory facilitation are not known.

The transcription factor cAMP-responsive element-binding protein (CREB) plays a key role in long-term memory formation (Silva et al., 1998). CREB phosphorylation at Ser133 enhances CREB-mediated gene transcription of target genes and the expression of these genes was shown to facilitate long-term memory and/or long-term potentiation (LTP), such as c-fos and zif268 (Jones et al., 2001; Fleischmann et al., 2003). In addition, CREB knock-out mice show impaired long-term memory and LTP (Bourtchuladze et al., 1994). A more recent study showed that CREB glycosylation impairs CREB-mediated transcription and memory formation (Rexach et al., 2012). In addition to CREB phosphorylation and CREB glycosylation, an earlier study showed that CREB could be SUMO modified in response to hypoxia and that overexpression of SUMO1 stabilizes CREB and enhances CREB-dependent gene reporter activity under hypoxia (Comerfold et al., 2003). These results suggest that SUMOylation is another posttranslational modification that occurs to CREB. However, whether CREB SUMOylation also takes place in the 
brain or if it is involved in memory formation is not known. Because PIAS1 is a transcriptional regulator that possesses small ubiquitin-like modifier (SUMO) E3 ligase activity (Kahyo et al., 2001), in the present study, we investigated whether CREB could be SUMO modified by PIAS1 and if CREB SUMOylation is another important molecular mechanism that mediates long-term memory formation.

\section{Materials and Methods}

Animals. Adult male Sprague Dawley rats (250-350 g) bred at the Animal Facility of the Institute of Biomedical Sciences, Academia Sinica in Taiwan were used. Animals were housed in a room maintained on a $12 \mathrm{~h} / 12$ $\mathrm{h}$ light/dark cycle (lights on at 6:30 A.M.) with food and water continuously available. Experimental procedures followed the National Institutes of Health's Guide for the Care and Use of Laboratory Animals.

Drugs. NMDA was purchased from Tocris Bioscience. Cycloheximide was purchased from Sigma-Aldrich. NMDA was injected into the rat CA1 area bilaterally at $0.7 \mu \mathrm{l}$ each side. The injection rate was $0.1 \mu \mathrm{l} / \mathrm{min}$.

Plasmid DNA construction. For construction of the Flag-tagged pias 1 plasmid, full-length pias 1 was cloned by amplifying the rat hippocampal pias1 cDNA with primers 5'-ATCGGGATCCCATGGCGGACAGTGC GGAAC-3' (forward) and 5'-ATCGGAATTCTCAGTCCAACGAGA TAATG-3' (reverse). The PCR product was subcloned between the BamHI and EcoRI sites of the mammalian expression vector pCMVTag2A (Invitrogen). Flag-PIAS1W372A, the ligase-deficient PIAS1 mutant, was generated using a site-directed mutagenesis kit (Stratagene). The same method was adopted for cloning the Flag-tagged pias 2 plasmid, Flag-tagged pias 3 plasmid, and Flag-tagged pias4 plasmid. The primers used for cloning pias2 were 5'-ATCGGAATTCGATGGCGGATTTCG AGGAG-3' (forward) and 5'-ATCGCTCGAGTCACTGTTGCACAGT ATC-3' (reverse). The primers used for cloning pias3 were $5^{\prime}$-ATC GGAATTCGATGGCGGAGCTGGGCG-3' (forward) and 5' -ATCGCT CGAGTCAGTCCAAGGAAATG-3' (reverse). The primers used for cloning

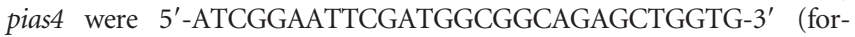
ward) and $5^{\prime}$-ATCGCTCGAGTCAGCAAGCGGGCACCAG-3' (reverse). The PCR products for PIAS2, PIAS3, and PIAS4 were subcloned between the EcoRI and XhoI sites of the mammalian expression vector pCMVTag2A. For construction of the His-PIAS1 plasmid, full-length pias1 was cloned by amplifying the Flag-PIAS1 plasmid with primers 5-ATGCGAATTCATGGCGGACAGTGCGGAAC-3 (forward) and 5-ATGCGCGGCCGCGTCCAACGAGATAATG-3 (reverse). The PCR product was subcloned between the EcoRI and NotI sites of the bacterial expression vector pET-32a (Novagen). For construction of the Flagtagged $U b c 9$ plasmid, full-length $U b c 9$ was cloned by amplifying the rat hippocampal $U b c 9$ cDNA with primers 5 '-ATCGGGATCCCATGTC GGGGATTGCCCTC- $3^{\prime}$ (forward) and $5^{\prime}$-ATCGGAATTCTTATGA GGGGGCAAACTTC-3' (reverse). The PCR product was subcloned between the BamHI and EcoRI sites of the mammalian expression vector pCMV-Tag2A. For construction of the Flag-tagged Pc2 plasmid, fulllength $P c 2$ was cloned by amplifying the rat hippocampal $P c 2 c D N A$ with primers 5'-ATCGGAATTCGATGGAGCTGCCAGCTGTTG-3' (forward) and 5'-ATCGCTCGAGCTACACCGTCACGTATTC-3' (reverse). The PCR product was subcloned between the EcoRI and XhoI sites of the mammalian expression vector $\mathrm{PCMV}-\mathrm{Tag} 2 \mathrm{~A}$. For construction of the Flag-tagged Ranbp2 $(\Delta \mathrm{FG})$ plasmid, partial-length Ranbp2 (aa 2553-2838) was cloned by amplifying the human kidney Ranbp2 with primers 5 '-ATGCGAATTCTTGAAAAGTAACAATAG-3' (forward) and 5' -ATGCCTCGAGAACTATCTTGCTTTCC-3' (reverse). The PCR product was subcloned between the EcoRI and XhoI sites of the mammalian expression vector pCMV-Tag2B. For construction of the V5tagged creb1 $\Delta$ plasmid, full-length creb1 $\Delta$ was cloned by amplifying the rat hippocampal crebl $1 \Delta$ cDNA with primers 5 '-ATTTGCGGCCGCCA CCATGACCATGGACTCTGGAGC-3' (forward) and 5'-GCTCTAGA TAATCTGACTTGTGGCAGTAAAGGTCC-3' (reverse). The PCR product was subcloned between the NotI and XbaI sites of the mammalian expression vector pcDNA3.1-V5-His (Invitrogen). For construction of the His-crebl $1 \Delta$ plasmid, full-length creb1 $1 \Delta$ was cloned by amplifying the V5-creb1 $\Delta$ plasmid with primers 5'-ATGCGAATTCATGACCATGG
ACTCTGGAG-3' (forward) and 5'-ATGCGCGGCCGCATCTGACTT GTGGCAGTAAAG-3' (reverse). The PCR product was subcloned between the EcoRI and NotI sites of the bacterial expression vector pET$32 \mathrm{a}$ (Novagen). For construction of the Flag-tagged creb1 $\Delta$ plasmid, fulllength creb $1 \Delta$ was cloned by amplifying the rat hippocampal V5-creb1 plasmid with primers $5^{\prime}$-ATCGGGATCCCATGACCATGGACTCTG GAGC-3' (forward) and 5'-ATCGAAGCTTATCTGACTTGTGGCAG TAAAG-3' (reverse). The PCR product was subcloned between the BamHI and HindIII sites of the mammalian expression vector pCMVTag2A. The CREB SUMOylation mutant and the phosphorylation mutant were generated with a site-directed mutagenesis kit (Stratagene). For construction of the creb double SUMOylation mutant, site-directed mutagenesis was done twice. For construction of the Myc-tagged sumo1 plasmid, full-length sumo1 was cloned by amplifying the mouse hippocampal sumo1 cDNA with primers $5^{\prime}$-GCAACCCGGGTGTCTGA CCAGGAGGCAAAACCTTC-3' (forward) and 5'-GCAAGGTACCCT AAACCGTCGAGTGACCCCCCGT-3' (reverse). The PCR product was cloned into the mammalian expression vector pCMV-Myc. The sumo1 $\Delta$ GG mutant was generated using a site-directed mutagenesis kit. For construction of GFP-2A-CREB1 $\Delta$ lentivitral vector, full-length creb1 $\Delta$ was cloned by amplifying the rat hippocampal Flag-creb $1 \Delta$ plamid with primers $5^{\prime}$-ATGCGAATTC GATTACAAGGATGACGAC-3' (forward) and 5'-ATGCGCGGCCGCATCTGACTTGTG GCAGTA-3' (reverse). The PCR product was subcloned between the EcoRI and NotI sites of the lentiviral vector pLenti-Tri-cistronic (ABM). GFP was cloned by amplifying the GFP gene from pLenti-CMV-GFP-2A-Puro-Blank $(\mathrm{ABM})$ and then subcloned into the pLenti-Tri-cistronic vector between the ScaI and KpnI sites upstream of the $2 \mathrm{~A}$ peptide (a self-processing viral peptide bridge) and creb1 $\Delta$ sequences. For construction of the GFP-2Acreb1 $1 \Delta$-sumol fusion lentivitral vector, the previously cloned Myc-sumo 1 plasmid was used as a template and the SUMO1 sequence was amplified with primers 5'-ATCGCTCGAGATGTCTGACCAGGAGGCAAAACC-3' (forward) and $5^{\prime}$-ATGCGGGCCCAACCGTCGAGTGACCCCCCGTT TG-3' (reverse). The PCR product was subcloned between XhoI and ApaI sites downstream of the CREB sequence from the previously cloned Flag-creb1 $1 \Delta$ plasmid. This Flag-creb1 $1 \Delta$-sumo1 fusion plasmid was then used as a template and the creb1 $\Delta$-sumol sequence was amplified with primers 5'-ATCGGAATTCATGACCATGGACTCTGGAGC-3' (forward) and $5^{\prime}$-ATCGGCGGCCGCAACCGTCGAGTGACCCCCCGTTTG-3' (reverse). The GFP-2A-creb1 $\Delta$ lentivitral vector was then substituted with the GFP-2A-creb1 $\Delta$-sumo1 lentiviral vector at EcoRI and NotI sites.

His-PIAS1 and His-CREB fusion protein purification. Bacterial culture of Rosetta-gami 2 (DE3) pLysS (Novagen) transformed with His-PIAS1 or His-CREB plasmids was grown at $37^{\circ} \mathrm{C}$ overnight in Overnight Express Instant LB Medium (Novagen). The His-PIAS1 pellet was resuspended and sonicated by lysis buffer $\left(50 \mathrm{mM} \mathrm{Na}_{2} \mathrm{HPO}_{4}, \mathrm{pH} 8.0,0.3 \mathrm{M}\right.$ $\mathrm{NaCl}, 8 \mathrm{~m}$ urea, $20 \mathrm{~mm}$ imidazole, $10 \mu \mathrm{g} / \mathrm{ml}$ PMSF, $2 \mu \mathrm{g} / \mathrm{ml}$ aprotinin, 2 $\mu \mathrm{g} / \mathrm{ml}$ leupeptin, and $2 \mu \mathrm{g} / \mathrm{ml}$ pepstatin). After centrifugation, the supernatant was added with His-Bind Resin beads (Ni-charged; Novagen) and incubated overnight with slow, end-over-end mixing. The HisPIAS1 proteins on beads were washed 3 times with washing buffer $(50 \mathrm{mM}$ $\mathrm{Na}_{2} \mathrm{HPO}_{4}, \mathrm{pH} 8.0,0.5 \mathrm{M} \mathrm{NaCl}, 8 \mathrm{M}$ urea, $20 \mathrm{~mm}$ imidazole) and protein refolding was reached by gradually decreasing the urea concentration in the washing buffer. The His-CREB pellet was resuspended in Bugbuster protein extraction reagent (Novagen) for protein extraction according to the instruction manual. Proteins on beads were eluted with $0.5 \mathrm{~m}$ imidazole in elution buffer (20 mm Tris- $\mathrm{HCl}, \mathrm{pH} 7.5,100 \mathrm{~mm} \mathrm{NaCl})$ and further dialyzed in $1 \mathrm{~L}$ of dialysis buffer (50 mM Tris- $\mathrm{HCl}, \mathrm{pH} 7.5,5 \mathrm{~mm} \mathrm{MgCl}_{2}$ ).

In vitro SUMOylation assay. The in vitro SUMOylation assay was performed in a total volume of $20 \mu \mathrm{l}$ containing $20 \mathrm{ng}$ of E1 enzyme (SAE1/ SAE2; Enzo Life Sciences), 200 ng of E2 enzyme (Ubc9), $3 \mu \mathrm{g}$ of SUMO1 protein, $3 \mu \mathrm{g}$ of CREB $1 \Delta$ protein, $2 \mathrm{~mm}$ ATP (Enzo Life Sciences), $500 \mathrm{ng}$ of PIAS1 protein, and $1 \mu \mathrm{g}$ of SENP1 enzyme (Enzo Life Sciences) in SUMOylation buffer (Enzo Life Sciences; Fig. 1B). Reactions were incubated at $30^{\circ} \mathrm{C}$ for $3 \mathrm{~h}$, stopped by adding sample buffer, and products were examined by SDS-PAGE separation. For in vitro SUMOylation assays performed in brain tissues after plasmid transfection or lentiviral vector transduction, endogenous CREB SUMOylation was determined by immunoprecipitation (IP) of CREB, followed by the addition of E1, 
A

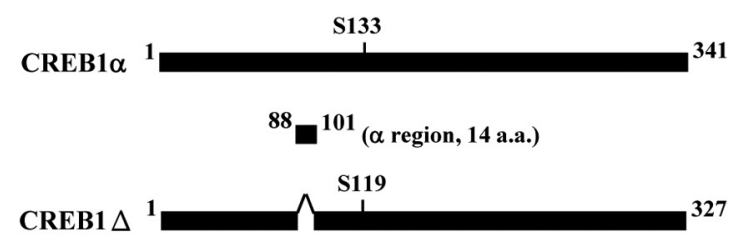

B

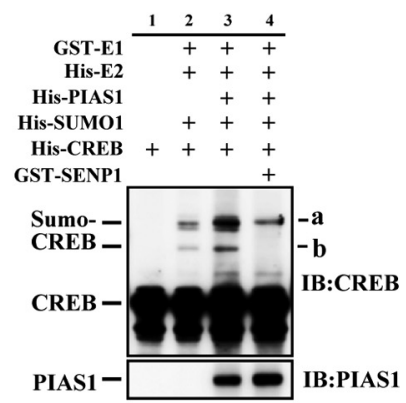

D

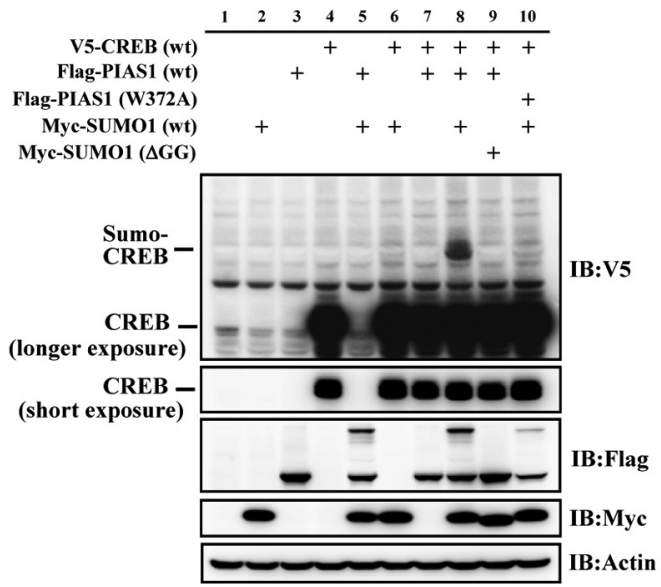

C
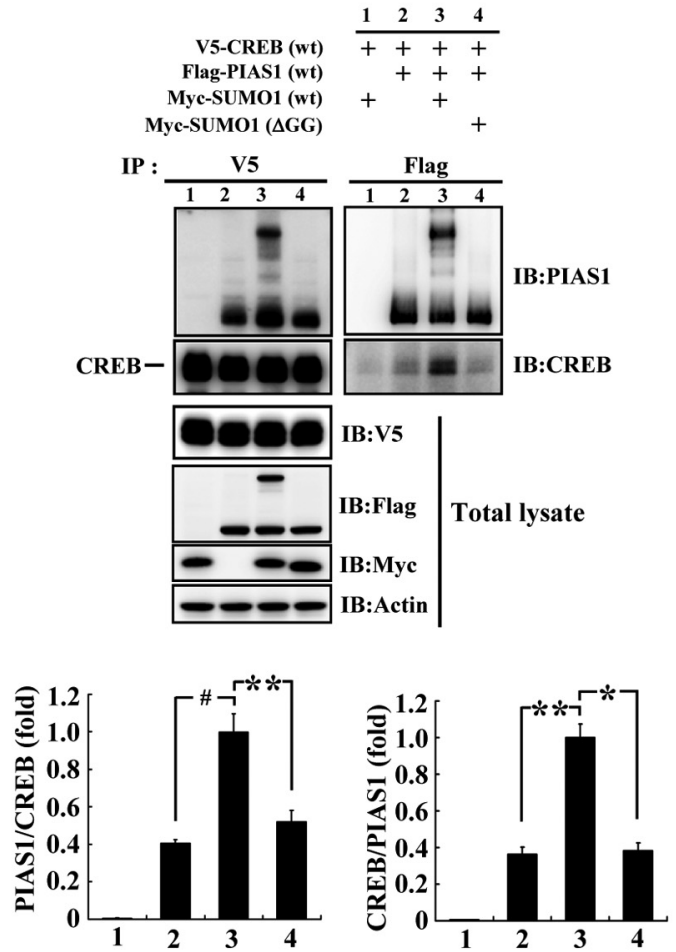

E

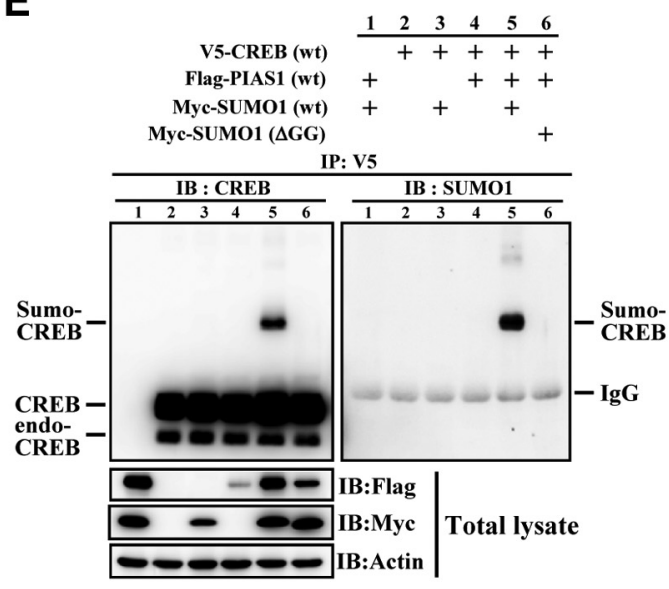

Figure 1. CREB is sumoylated by PIAS1 in vitro and in HEK293T cells. $A$, Alignment and comparison of CREB $1 \alpha$ and CREB1 $\Delta$ isoforms showing that CREB1 $\Delta$ is 14 aa shorter than that of CREB1 $\alpha$ (aa 88 -101), and that Ser119 of CREB1 $\Delta$ corresponds to Ser133 of CREB1 $\alpha$. B, In vitro SUMOylation assay and Western blot showing PIAS1 SUM0ylation of CREB. "a" and "b" indicate the sumoylated form of the CREB bands. C, Co-IP experiment showing the association between PIAS1 and CREB in HEK293T cells with or without the cotransfection of Myc-SUM01 or Myc-SUM01 ( $\triangle$ GG). The quantified results are shown on the bottom. D, Transfection of different plasmids to HEK293T cells and Western blot showing PIAS1 SUMOylation of CREB in HEK293T cells. E, Confirmation of PIAS1 SUM0ylation of CREB in HEK293T cells by IP and Western blot against CREB and SUM01. endo-CREB, Endogenous CREB; $\triangle$ GG, C-terminal diglycine motif truncated SUM01. Data are expressed as mean \pm SEM. ${ }^{* *} p<0.05 ;{ }^{* *} p<0.01$; \#p $<0.001$. Experiments were performed in triplicate.

E2, and the recombinant PIAS1 protein to verify that CREB was actually sumoylated by the PIAS1 E3 ligase. The effect of different transfections and transductions on CREB SUMOylation was also determined. For all CREB SUMOylation assays performed in brain tissues after spatial training or PIAS1 siRNA transfection, endogenous CREB SUMOylation was determined by IP of CREB, followed by in vitro SUMOylation assay without the addition of E1, E2, and the recombinant PIAS1 protein. No recombinant CREB protein was added to the SUMOylation reaction in the present study.

Plasmid transfection to HEK293T cells. Human embryonic kidney 293T (HEK293T) cells were cultured in Dulbecco's Modified Eagle's Medium (DMEM) (HyClone) supplemented with 10\% heat-inactivated fetal bovine serum (FBS) (HyClone), 100 units $/ \mathrm{ml}$ penicillin, and $100 \mu \mathrm{g} / \mathrm{ml}$ streptomycin (Invitrogen) at $37^{\circ} \mathrm{C}$ in a $5 \% \mathrm{CO}_{2}$ incubator. HEK293T cells were seeded in a 6-well (for the IP experiment) or 12-well culture plate overnight and cotransfected with the mixture containing a total of $1.6 \mu \mathrm{g}$ of plasmids and 4 $\mu \mathrm{l}$ of Lipofectamine 2000 (Invitrogen) according to the manufacturer's protocols. Forty-eight hours after plasmid transfection (or cotransfection), cells were washed 3 times with ice-cold phosphate buffered saline (PBS) and lysed with lysis buffer (25 mм HEPES, pH 7.6, $150 \mathrm{~mm} \mathrm{NaCl} 5$ mm EDTA, pH 8.0, $20 \mathrm{~mm} N$-ethylmaleimide, $10 \%$ glycerol, $1 \%$ Triton X-100) containing a mixture of protease inhibitor and phosphatase inhibitor (Roche).

Lentivirus preparation. For lentivirus packaging, HEK293LTV cells (Cell Biolabs) were transfected with $1.5 \mu \mathrm{g}$ of psPAX2 (plasmid 12260; 
Addgene), $0.5 \mu \mathrm{g}$ of pMD2.G (plasmid 12259; Addgene), and $2 \mu \mathrm{g}$ of pLenti-GFP-2A-creb1 (WT or double SUMOylation mutant) or $2 \mu \mathrm{g}$ of pLenti-CMV-GFP-2A-Puro-Blank (ABM) coding for GFP as a control using $10 \mu \mathrm{l}$ of Lipofectamine 2000 (Invitrogen) in 6-well cell culture dishes. Lentiviral supernatant was harvested $48 \mathrm{~h}$ after transfection and again $12 \mathrm{~h}$ later and then kept at $4^{\circ} \mathrm{C}$ over the collecting period. The collected lentiviral supernatant was centrifuged at $2500 \times \mathrm{g}$ for $10 \mathrm{~min}$ and filtered through a $0.45 \mu \mathrm{m}$ syringe filter. The speedy lentivirus purification solution (ABM) was added to the filtrated lentiviral supernatant $(1: 9, \mathrm{v} / \mathrm{v})$ and mixed thoroughly by inversion. The lentiviral supernatant mixture was centrifuged directly at $5000 \times g$ for $10 \mathrm{~min}$ at $4^{\circ} \mathrm{C}$. The supernatant was decanted and the viral pellet was resuspended in $100 \mu \mathrm{l}$ of culture medium (DMEM with 10\% FBS). The lentivirus titer was determined using the lentivirus qPCR Titer Kit (ABM) according to the manufacturer's protocols $(\mathrm{ABM})$. The stock concentration for the lentivirus vector was $5 \times 10^{7}$, the concentration for the lentivirusCREB $1 \triangle$ WT vector was $3.5 \times 10^{7}$, and the concentration for the lentivirus-CREBK1 $\Delta 271$ RK290R was $6.9 \times 10^{7}$. For long-term storage of the lentiviral particle, the final viral stock was stored at $-80^{\circ} \mathrm{C}$ in aliquots.

Intra-hippocampal lentiviral vector transduction. For the water maze learning experiment that requires longer time expression of DNA in the $\mathrm{CA} 1$ area, lentiviral vector injection was adopted. Animals were anesthetized with pentobarbital (40 mg/kg, i.p.) and subjected to stereotaxic surgery. Two 23 gauge, stainless steel, thin wall cannulae were implanted bilaterally to the hippocampal CA1 area at the following coordinates: 3.5 $\mathrm{mm}$ posterior to the bregma, $2.5 \mathrm{~mm}$ lateral to the midline, and $3.4 \mathrm{~mm}$ ventral to the skull surface. After animals recovered from the surgery, the lentiviral vector of GFP, CREB1 $\Delta W T$, or CREB1 $\Delta$ K271RK290R (the CREB1 $\Delta$ double SUMOylation mutant) was injected into the CA1 area bilaterally. Before injection, all lentiviral vectors were diluted in PBS, $\mathrm{pH}$ 7.4 , to a titer of $3.5 \times 10^{7} / \mu$ l. The volume for lentiviral vector injection was $0.7 \mu \mathrm{l}$ each side. The injection rate was $0.1 \mu \mathrm{l} / \mathrm{min}$. Animals were subjected to water maze learning $10 \mathrm{~d}$ after lentiviral vector transduction.

Intra-hippocampal plasmid DNA transfection and siRNA interference. For experiments that only required transient plasmid DNA expression in the CA1 area, the method from that of a previous study (Abdallah et al., 1996) was adopted with some modification. The nonviral vector transfection reagent polyethyleneimine (PEI) was used because we have demonstrated previously that it does not produce toxicity to hippocampal neurons (Chao et al., 2011). Before injection, plasmid DNA was diluted in $5 \%$ glucose to a stock concentration of $2.77 \mu \mathrm{g} / \mu \mathrm{l}$. Branched PEI of 25 $\mathrm{kDa}$ (Sigma-Aldrich) was diluted to $0.1 \mathrm{M}$ concentration in $5 \%$ glucose. Immediately before injection, $0.1 \mathrm{M}$ PEI was added to the DNA solution $(0.45 \mu \mathrm{l}$ of PEI and $0.55 \mu \mathrm{l}$ of plasmid DNA) to reach a ratio of PEI nitrogen per DNA phosphate equal to 10. The final concentration of the plasmid DNA was $1.5 \mu \mathrm{g} / \mu \mathrm{l}$. For siRNA transfection, PIAS1 siRNA was diluted in distilled water and stored at a stock concentration of $20 \mathrm{pmol} /$ $\mu$ l. Before injection, $0.1 \mathrm{M}$ PEI was added to PIAS1 siRNA (1:1, v/v) and the final concentration of PIAS1 siRNA was $10 \mathrm{pmol} / \mu \mathrm{l}$. The sequences for PIAS1 siRNA were as follows: 5'-UCCGGAUCAUUCUAGAGCUTT-3' (sense) and 5'-AGCUCUAGAAUGAUCCGGATT-3' (antisense). The silencer negative control number 1 siRNA (control siRNA), siRNAs with sequences that do not target any gene product (Ambion), were used as a control. PIAS1 siRNA and control siRNA were synthesized from Ambion. The mixture was subjected to vortex for $30 \mathrm{~s}$ and allowed to equilibrate for $15 \mathrm{~min}$ before injection. The injection volume was $0.7 \mu \mathrm{l}$ on each side. The injection rate was $0.1 \mu \mathrm{l} / \mathrm{min}$. For PIAS1 siRNA and control siRNA transfections, 2 injections were made to the CA1 area in each rat during the $5 \mathrm{~d}$ water maze learning period, with 1 injection given $48 \mathrm{~h}$ before the first swim trial on the first day and the second injection given before the first swim trial on the second day. Animals were decapitated $48 \mathrm{~h}$ after plasmid DNA transfection or siRNA transfection or immediately after the probe trial test and their CA1 tissue was punched out for biochemical assays.

Western blot. The hippocampal CA1 tissue was lysed in RIPA buffer (50 mm Tris- $\mathrm{HCl}$, pH 7.4, $150 \mathrm{~mm} \mathrm{NaCl}$, and 1\% IGEPAL CA-630 with mixture of protease inhibitor and phosphatase inhibitor). The lysate was resolved by $8 \%$ SDS-PAGE. The proteins resolved by SDS-PAGE were transferred onto the PVDF membrane (Millipore). Protein samples from HEK cells were separated on $8 \%$ or $12 \%$ SDS-PAGE and then transferred to nitrocellulose membranes (Sartorius). Immunoblotting was performed using rabbit anti-CREB (Cell Signaling Technology), anti-pS133CREB (Cell Signaling Technology), anti-PIAS1 (Epitomics), anti-SUMO1 (Cell Signaling Technology), and mouse anti-V5 (Serotec), anti-Flag M2 (SigmaAldrich), anti-EGFP (Sigma-Aldrich), anti-Myc (SAB), and anti-actin (Millipore) antibodies. Because both CREB1 $\alpha$ and CREB1 $\Delta$ (14 aa shorter; Fig. $1 A$ ) are present in the brain with CREB1 $\Delta$ as the predominant isoform identified (Blendy et al., 1996) and the neighboring 36 aa (at least 18 aa toward the $5^{\prime}$ end and $>18$ aa toward the $3^{\prime}$ end) from Ser 133 of CREB $1 \alpha$ and Ser 119 of CREB $1 \Delta$ are identical (Fig. $1 A$ ), it is conceivable that the pCREB antibody recognizes both endogenous Ser133 of CREB $1 \alpha$ and Ser119 of CREB1 $\Delta$. The secondary antibody used was horseradish peroxidase (HRP)-conjugated goat-anti rabbit or goat-anti mouse IgG antibody (Jackson ImmunoResearch). Protein expression was detected by chemiluminescence HRP substrate (Millipore) and was exposed to the ImageQuant LAS 4000 mini image system (GE Healthcare) for visualization of the protein bands. These bands were quantified using ImageJ software.

IP and co-IP. For IP of V5-CREB or co-IP of Flag-PIAS1 and V5-CREB in HEK293T cells, cells were cotransfected with the indicated plasmids and lysed $48 \mathrm{~h}$ later. The lysates $(0.5 \mathrm{mg})$ were immunoprecipitated with $1 \mu \mathrm{l}$ of mouse anti-V5 antibody (Serotec) or mouse anti-Flag M2 antibody (Sigma-Aldrich) at $4^{\circ} \mathrm{C}$ for $2 \mathrm{~h}$ and added with $20 \mu \mathrm{l}$ of ( $10 \%$ slurry) Protein G Mag Sepharose Xtra beads (GE Healthcare) overnight. For Co-IP of PIAS1 and CREB in the hippocampal tissue, the clarified hippocampal lysate $(1 \mathrm{mg})$ was immunoprecipitated with $1 \mu \mathrm{l}$ of rabbit anti-IgG (Cell Signaling Technology) and $2 \mu$ l of rabbit anti-CREB antibody (Cell Signaling Technology) or rabbit anti-PIAS1 antibody (Epitomics) at $4^{\circ} \mathrm{C}$ for $2 \mathrm{~h}$ and added with $20 \mu \mathrm{l}$ of ( $10 \%$ slurry) Protein A Mag Sepharose Xtra beads (GE Healthcare) overnight. The immune complex on beads were washed with PBS three times and boiled in sample buffer. The immunoprecipitated products were subjected to $8 \%$ SDS-PAGE, transferred onto the membrane, and immunoblotted with the indicated antibody.

IP and in vitro SUMOylation assay in CA1 tissue. To analyze the endogenous PIAS1 SUMO E3 ligase activity, IP of PIAS1 as the source of E3 was performed for in vitro SUMOylation assay. Hippocampal CA1 tissue lysate was prepared in the same way as that prepared for Western blot. For IP PIAS1, the clarified lysate $(0.5 \mathrm{mg})$ was immunoprecipitated with $3 \mu \mathrm{l}$ of anti-PIAS1 antibody (Epitomics) at $4^{\circ} \mathrm{C}$ for $3 \mathrm{~h}$. The Protein A Mag Sepharose Xtra beads ( $20 \mu \mathrm{l}, 50 \%$ slurry; GE Healthcare) were added to the IP reaction product to catch the immune complex at $4^{\circ} \mathrm{C}$ overnight. The immune complex on beads was washed three times with $\mathrm{PBS}$ and subjected to in vitro SUMOylation reaction. In vitro SUMOylation reaction was performed with or without the addition of $3 \mu \mathrm{l}$ of recombinant PIAS1 protein (Abnova), $1 \mu \mathrm{l}$ of E1, and $1 \mu \mathrm{l}$ of E2 proteins. In vitro SUMOylation assay was performed using the SUMO link kit according to the manufacturer's instructions (Active Motif). The reaction mixture was boiled in Laemmli sample buffer at $95^{\circ} \mathrm{C}$ for $10 \mathrm{~min}$ and the in vitro SUMOylation product was subjected to $8 \%$ SDS-PAGE, followed by transferring onto the nitrocellulose membrane (Sartorius). The membrane was immunoblotted with rabbit anti-PIAS1 (Epitomics) or rabbit anti-CREB (Cell Signaling Technology) antibody.

Biotinylated oligonucleotides pull-down assay for CREB DNA-binding activity. DNA oligonucleotides containing two CRE elements (underlined; 5' AGA GAT TGC CTG ACG TCA GAG AGC TAG GAT TGC CTG ACG TCA GAG AGC TAG 3' for the sense strand and 5' CTA GCT CTC TGA CGT CAG GCA ATC CTA GCT CTC TGA CGT CAG GCA ATC TCT 3 ' for the antisense strand) were conjugated with a $5^{\prime}$ biotin on the sense strand according to the method described previously (Vallejo et al., 1992). Both complementary oligonucleotides were resuspended in the annealing buffer (10 mm Tris, $\mathrm{pH}$ 8.0, $50 \mathrm{~mm} \mathrm{NaCl}, 1 \mathrm{~mm}$ EDTA). For annealing the sense and antisense oligonucleotides, $10 \mu \mathrm{l}$ each of the complementary oligonucleotides, together with $80 \mu \mathrm{l}$ of the annealing buffer, were mixed in a $0.5 \mathrm{ml}$ microtube that was placed in a heating block at $90^{\circ} \mathrm{C}$. The heating block was allowed to gradually cool down to room temperature and stored on ice or at $-20^{\circ} \mathrm{C}$ until use. For the CREB 
pull-down assay, the clarified hippocampal CA1 tissue lysate $(0.4 \mathrm{mg})$ was added with $6 \mu \mathrm{l}$ of duplex oligonucleotides (100 $\mu \mathrm{M}$ ) and poly dI-dC (1 $\mu \mathrm{g} / \mathrm{ml}$; GE Healthcare) at $4^{\circ} \mathrm{C}$ overnight. The streptavidin agarose beads (10 $\mu$; Sigma-Aldrich) were added to the pull-down reaction product to catch the CREB-DNA oligonucleotide complex at $4^{\circ} \mathrm{C}$ for $3 \mathrm{~h}$. The pull-down reaction complex on beads was then washed three times with PBS and boiled in Laemmli sample buffer at $95^{\circ} \mathrm{C}$ for $10 \mathrm{~min}$. For analysis of CREB DNA-binding activity, the pull-down assay product was subjected to $8 \%$ SDS-PAGE, followed by transferring onto the PVDF membrane (Millipore) and immunoblotted with anti-CREB antibody (Cell Signaling Technology).

Chromatin immunoprecipitation assay. The chromatin immunoprecipitation (ChIP) assay was performed according to the protocol of the Millipore ChIP assay kit (catalog \#17-10085). Briefly, rats were transfected with Flag-vector, Flag-creb1 $\Delta W T$, and Flag-creb1 $\Delta$ K271RK290R (double SUMOylation mutant) plasmid to the CA1 area and their CA1 tissues were collected for ChIP assay. The CA1 tissues were then fixed with $1 \%$ formaldehyde at room temperature for $15 \mathrm{~min}$. After adding glycine for $5 \mathrm{~min}$ incubation to quench the unreacted formaldehyde, the tissues were washed with PBS. The tissues were collected and changed to cell lysis buffer plus protease inhibitor mixture II, then homogenized with a glass Dounce homogenizer. After centrifugation, the pellet was placed to nuclear lysis buffer plus protease inhibitor mixture II for sonication. The chromatin was immunoprecipitated using Flag M2 monoclonal antibody (Sigma-Aldrich) and mouse IgG antibody (Millipore). DNA purified from the immunoprecipitated samples was subjected to PCR with the Eppendorf Mastercycler Gradient PCR system. The primers for $b d n f$ promoter were designed according toa previous study (Tian et al., 2010). The forward primer was as follows: 5'-AGCTCTTGCAGACTAAATCGTGAGTTT-3' and the reverse primer was as follows: $5^{\prime}$-CAAGAACCTGGTGTTGAGCTCATATTT-3'. The PCR product was $151 \mathrm{bp}$ in length for $b d n f$ and was separated by agarose gel electrophoresis.

ChIP quantitative real-time PCR. For accurate determination of levels of specific chromatin DNA in ChIP samples, real-time PCR analysis was performed using the Rotor-Gene Q real-time PCR cycler with iQ SYBR Green Supermix (Bio-Rad) according to the instruction manual (QIAGEN). The primers for the $b d n f$ promoter were designed according to a previous study (Lubin et al., 2008). The forward primer was as follows: 5'-GTGGAGGAGAGGTGCCTTTTGA-3' and the reverse primer was as follows: $5^{\prime}$-GTCCTCTGGGGACCATTTACCC-3'. The cycle threshold $\left(C_{\mathrm{t}}\right)$ value and data were analyzed using Rotor-Gene Q detection software (QIAGEN). The relative binding activity (fold) was determined using the $2^{-(\Delta \Delta \mathrm{Ct})}$ method (Livak and Schmittgen, 2001).

Quantitative real-time PCR. For determination of the $b d n f$ mRNA level, total RNA from the CA1 tissue was isolated using the RNAspin mini kit (GE Healthcare). Total RNA ( $1 \mu \mathrm{g})$ was reverse transcribed using the Transcriptor First Strand cDNA Synthesis Kit (Roche). Real-time PCR was performed using the Rotor-Gene Q real-time PCR Cycler (QIAGEN) with iQ SYBR Green Supermix (Bio-Rad). The PCR parameters used for $b d n f$ and $G A P D H$ were as follows: $95^{\circ} \mathrm{C}$ for $10 \mathrm{~min}, 95^{\circ} \mathrm{C}$ at $10 \mathrm{~s}$ for 40 cycles, followed by $60^{\circ} \mathrm{C}$ for $45 \mathrm{~s}$. The sequence for the forward primer of $b d n f$ (exon 4) was as follows: 5'-GTGACAACAATGTGACTCCACT-3' and that for the reverse primer was as follows: $5^{\prime}$-GCCTTCATGCAACCG AAGTA-3'. The sequence for the forward primer of GAPDH was as follows: $5^{\prime}$-GGCAAGTTCAATGGCACAGT- $3^{\prime}$ and that for the reverse primer was as follows: 5'-TGGTGAAGACGCCAGTAGACTC-3'. Data were analyzed using Rotor-Gene Q Detection Software (QIAGEN) and the expression level (fold) was determined using the $2^{-(\Delta \Delta \mathrm{Ct})}$ method (Livak and Schmittgen, 2001).

Immunohistochemistry. Rats were anesthetized with pentobarbital (40 $\mathrm{mg} / \mathrm{kg}$, i.p.) and perfused with PBS, followed by $4 \%$ paraformaldehyde. Brains were removed and postfixed in a $20 \%$ sucrose $/ 4 \%$ paraformaldehyde solution for 20-48 h. Brains were then frozen, cut into $30 \mu \mathrm{m}$ sections on a cryostat, and mounted on gelatin-coated slides. Brain sections were rinsed with $1 \times$ PBS for $10 \mathrm{~min}$ and mounted with DAPIFluoromount-G (Southern Biotech). For immunofluorescence visualization of crebl $1 \Delta$ plasmid transfection and expression, EGFP-tagged creb1 $\Delta W T$ plasmid was transfected to the CA1 area and brain sections were prepared
$48 \mathrm{~h}$ after EGFP-tagged creb1 $\Delta W T$ transfection for visualization of EGFP fluorescence by a confocal microscope. Digital photomicrographs were also taken.

Water maze learning. The water maze used for rats was a plastic, circular pool $2.0 \mathrm{~m}$ in diameter and $0.6 \mathrm{~m}$ in height that was filled with water $(25 \pm$ $2^{\circ} \mathrm{C}$ ) to a depth of $20 \mathrm{~cm}$. A circular platform $(13 \mathrm{~cm}$ in diameter $)$ was placed at a specific location away from the edge of the pool. The top of the platform was submerged $1.5 \mathrm{~cm}$ below the water surface. Water was made cloudy by adding milk powder. Distinctive visual cues were set on the wall.

For spatial learning and training, animals were subjected to three trials a day with one given in the morning, one given in the early afternoon, and the other one given in the late afternoon. The training procedure lasted for $4 \mathrm{~d}$ and a total of 12 trials were given. For each trial, the animal was placed at different starting position spaced equally around the perimeter of the pool in a random manner. Animals were given $60 \mathrm{~s}$ to find the platform. If an animal could not find the platform, it was guided to the platform and allowed to stay there for $20 \mathrm{~s}$. The time that each animal took to reach the platform was recorded as the escape latency. A probe trial of $60 \mathrm{~s}$ was given $2 \mathrm{~d}$ after the end of training to test memory retention. For the probe trial test, animals were placed in the pool with the platform removed and the time they spent in each quadrant [target quadrant (T), left quadrant (L), opposite quadrant (O), and right quadrant $(\mathrm{R})$ ] and their swimming paths were recorded.

For spatial training in naive rats, animals were subjected to watermaze learning for 3 trials a day for a total of 3 trials ( $1 \mathrm{~d}), 6$ trials $(2 \mathrm{~d})$, or 15 trials $(5 \mathrm{~d})$ and no probe trial was given. Animals in the swimming control group swam for the same amount of time for each trial except that the visual cues and the platform were not present. Animals were decapitated at the end of training and their CA1 tissue was punched out for biochemical determinations.

Statistics. Water maze data were analyzed by repeated-measures ANOVA, followed by post hoc Newman-Keuls multiple comparisons (represented by $q$ value). Probe trial data and biochemical data were analyzed with one-way ANOVA followed by Newman-Keuls multiple comparisons (for more than two groups) or with Student's $t$ test (for two groups).

\section{Results}

\section{CREB is sumoylated by PIAS1 in vitro and in HEK293T cells and CREB is associated with PIAS1 in HEK293T cells}

Alignment and comparison of CREB $1 \alpha$ and CREB1 $\Delta$ isoforms showed that CREB1 $\Delta$ is 14 aa shorter than CREB $1 \alpha$ (from aa 88 to 101$)$ and that Ser119 of CREB1 $\Delta$ corresponds to Ser133 of CREB1 $\alpha$ (Fig. 1A). Because CREB1 $\Delta$ is the major isoform we obtained from the rat hippocampus and it is also the predominant isoform found in the brain (Blendy et al., 1996), we used it for the present study. To address the issue of CREB SUMOylation by PIAS1, we first performed the in vitro SUMOylation assay. His-tagged E2, His-tagged PIAS1, His-tagged SUMO1, Histagged CREB or GST-tagged E1, or GST-tagged SENP1 recombinant proteins were added to the reaction and Western blots against CREB and PIAS1 were performed. Results revealed that a basal level of CREB SUMOylation was observed when E1, E2, SUMO1, and CREB proteins were added, but the addition of the PIAS1 protein apparently enhanced the SUMOylation of CREB and this effect was apparently blocked by the addition of sentrinspecific protease 1 (SENP1), an enzyme that removes sumo from sumo-conjugated protein (Fig. $1 B$ ). There are two major sumoCREB bands in Figure $1 B$ (designated as " $a$ " and "b"). According to molecular weight, the lower band probably indicates that a single SUMO1 molecule is conjugated to the CREB protein, whereas the upper band may indicate that two SUMO1 molecules are conjugated to the CREB protein at different SUMO acceptors on CREB. The reason that SENP1 did not completely abolish CREB SUMOylation is probably that the concentration of SENP1 added was not high enough or there was a certain degree of degradation of the SENP1 enzyme activity during experimentation. 
Next, we investigated whether PIAS1 is associated with CREB in the cells. V5-CREB, Flag-PIAS1, Myc-SUMO1, and MycSUMO1 $(\Delta \mathrm{GG})$ plasmids in different combinations were transfected to HEK293T cells and IP against these tags, followed by Western blots against PIAS1 and CREB, were performed. Results revealed that PIAS1 is associated with CREB endogenously and this association was enhanced when Myc-SUMO1 was cotransfected (Fig. 1C, left). Under this condition, PIAS1 itself was also sumoylated (Fig. 1C, right). However, the association between PIAS1 and CREB was prevented by cotransfection of MycSUMO1 ( $\Delta \mathrm{GG})$, the SUMO1 plasmid that lacks the C-terminal diglycine motif essential for SUMO 1 conjugation (Ihara et al., 2005). The quantitative results of these comparisons from three independent experiments are shown at the bottom $\left(F_{(3,8)}=39.06, p<\right.$ 0.001 for ratio of PIAS1/CREB and $F_{(3,8)}=14.01, p<0.01$ for ratio of CREB/PIAS1). Immunoblotting against V5, Flag, and Myc tags in total lysate confirmed the transfection of plasmids and the expression of proteins (Fig. $1 C$, bottom). Next, we investigated whether CREB could be sumoylated by PIAS1 in the cell. V5-CREB, FlagPIAS1 (or Flag-PIAS1W372A), and Myc-SUMO1 (or MycSUMO1 $\Delta$ GG) plasmids were transfected to HEK293T cells and Western blots were performed. Results revealed that transfection of the V5-CREB, Flag-PIAS1, and Myc-SUMO1 plasmids yielded an apparent and specific band of CREB SUMOylation that was verified by Western blot against V5 (Fig. 1D) and confirmed by Western blot against CREB (Fig. 1E, left) and SUMO1 (Fig. 1E, right). However, transfection of Myc-SUMO1 $\Delta$ GG and Flag-PIAS1W372A both prevented CREB SUMOylation by PIAS1.

\section{Identification of the major SUMO acceptors on CREB}

We then determined the SUMOylation site on CREB by PIAS1. There were 13 lysine residues on the CREB protein in all of them, but no consensus SUMO-substrate motif $(\psi$-K-X-E, where $\psi$ stands for a hydrophobic amino acid) was found. Therefore, we have generated individual CREB sumo-mutants against these lysine residues. Each V5-tagged CREB lysine residue mutant construct was then cotransfected with Flag-PIAS1 and Myc-SUMO1 for identification of the target SUMOylation site on CREB by PIAS1. The first batch of results revealed that an apparent band of CREB SUMOylation was observed when V5-CREBWT, FlagPIAS1WT, and Myc-SUMO1WT were cotransfected, but the intensity of sumoylated CREB was diminished when the CREB sumo-mutant at Lys271 was transfected. The other four CREB sumo-mutants (K109R, K122R, K141R, and K278R) did not produce the same effect (Fig. 2A). The second batch of results revealed that, among the four other lysine residues examined (K289R, K290R, K291R, and K295R), only transfection of the CREB sumo-mutant at Lys 290 diminished the intensity of CREB SUMOylation (Fig. 2B). The third batch of results revealed that, among the additional four lysine residues examined (K309R, K316R, K319R, and K325R), none affected CREB SUMOylation (Fig. 2C). We further confirmed the above two SUMOylation sites (Lys271 and Lys290) by transfection of the Flag-PIAS1WT plasmid (or Flag-PIAS1W372A), Myc-SUMO1, together with V5-CREBWT or V5-CREBK271R or V5-CREBK290R, or the double sumo-mutant V5-CREBK271RK290R plasmid to HEK293T cells and by Western blots against V5 and other tags. A representative gel pattern of CREB SUMOylation under these conditions is shown in Figure 2D, left. Statistical analyses from three independent experiments revealed an overall significant effect of these treatments on CREB SUMOylation $\left(F_{(7,16)}=133.58\right.$, $p<0.001)$. Further analyses indicated that CREB was consistently sumoylated by PIAS1 (Fig. 2D, left, lane 4 vs lane 3 ), but the intensity of CREB SUMOylation diminished when V5CREBK271R or V5-CREBK290R was transfected $(q=3.35, p<$ 0.05 and $q=9.69, p<0.001$, respectively, vs the V5-CREBWT group) and this band was no longer observed when the double sumo-mutant V5-CREBK271RK290R was transfected $(q=$ 13.79, $p<0.001$ vs the V5-CREBWT group; Fig. $2 D$, right). CREB SUMOylation was similarly blocked when FlagPIAS1W372A (instead of Flag-PIAS1WT) was transfected ( $q=$ 19.97, $p<0.001$ vs the V5-CREBWT group; Fig. $2 D$, right).

\section{PIAS1 SUMOylation of CREB increases the level of CREB expression}

We then investigated whether PIAS1 SUMOylation of CREB affects the expression level of CREB. V5-CREB, Flag-PIAS1, and Myc-SUMO1 plasmids were cotransfected to HEK293T cells with the administration of the translation inhibitor cycloheximide $(50 \mu \mathrm{g} / \mathrm{ml})$ for different time periods. Flag-PIAS1 was not transfected in the control group. Western blots against different tags were performed. The results revealed a similar degradation rate of the CREB protein for both groups, but cotransfection of Flag-PIAS1 increased the expression level of CREB (Fig. 2E). The quantified result at each time point examined is shown in Figure $2 E$, bottom. Plasmid transfection and expression was confirmed by Western blots against different tags (Fig. 2E, top). Moreover, the PIAS protein family consists of different members of the PIAS protein, including PIAS1, PIAS2, PIAS3, and PIAS4 (Rytinki et al., 2009). Next, we investigated whether other PIAS proteins also sumoylate CREB and if the E2 ligase Ubc9 and other E3 ligases also sumoylate CREB. V5-CREB and Myc-SUMO1 were cotransfected with different Flag-tagged PIAS plasmid or Flag-Pc2 (E3 ligase) plasmid, Flag-RanBP2 ( $\Delta \mathrm{FG}$; E3 ligase) plasmid, or FlagUbc9 (E2 ligase) plasmid to HEK293T cells and Western blots against V5 and other tags were performed. RanBP2 ( $\Delta \mathrm{FG})$ represents the partial sequence of full-length RanBP2, which retained its E3 ligase activity (Pichler et al., 2002). A representative gel pattern of CREB SUMOylation under these conditions is shown in Figure $2 F$, left. Statistical analyses from three independent experiments revealed an overall significant effect of these manipulations on CREB SUMOylation $\left(F_{(7,16)}=145.42, p<0.001\right)$ and that all of the PIAS family proteins sumoylated CREB (Fig. $2 F$, left, lanes 3-6 vs lane 2). Further analyses indicated that PIAS1 and PIAS3 had a relatively stronger effect in SUMOylation of CREB than did PIAS2 and PIAS4 $(q=3.55, p<0.05$ and $q=$ 2.93, $p<0.05$; Fig. $2 F$, right). Other E3 ligases and the E2 ligase Ubc9 did not have such an effect (Fig. 2F, left, lanes 7-8 vs lane 3 and lane 2 vs lane 3 ).

\section{CREB is sumoylated by PIAS1 in the hippocampus and spatial training increases CREB SUMOylation}

We next investigated whether PIAS1 SUMOylation of CREB takes place in the hippocampus. The co-IP experiment for PIAS1 and CREB was first performed in the hippocampus and the results revealed that PIAS1 was associated with CREB and vice versa (Fig. 3A). Furthermore, the Flag-vector, Flag-CREBWT, FlagCREBK271R, Flag-CREBK290R, and Flag-CREBK271RK290R plasmids were transfected to the hippocampal CA1 area and in vitro SUMOylation assay was performed $48 \mathrm{~h}$ later. A representative gel pattern of CREB SUMOylation under these conditions is shown in Figure 3B, left. Statistical analyses from three independent experiments revealed an overall significant effect of these transfections on CREB SUMOylation $\left(F_{(5,12)}=17.55, p<\right.$ 0.001). Further analyses indicated that CREB SUMOylation by PIAS1 was enhanced when the Flag-CREBWT plasmid was trans- 
A

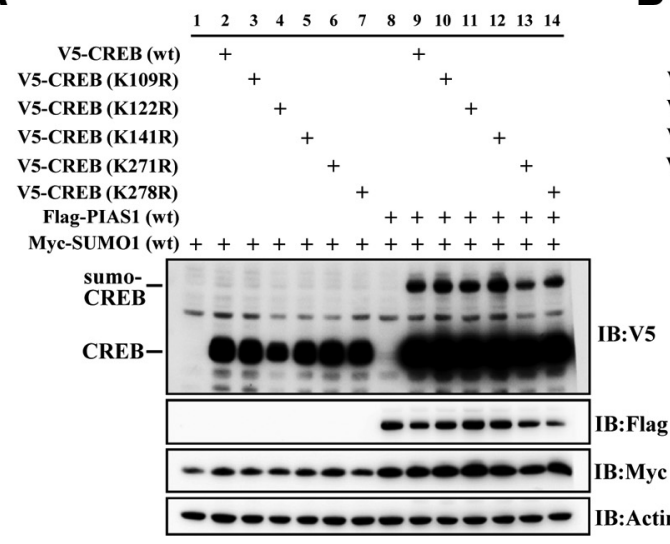

B

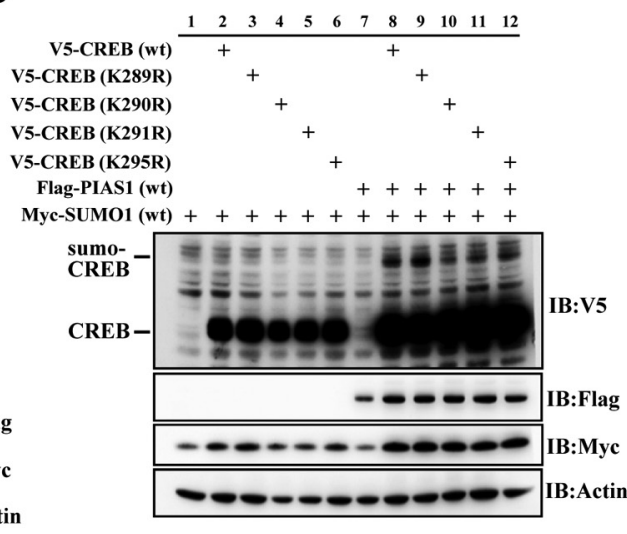

C

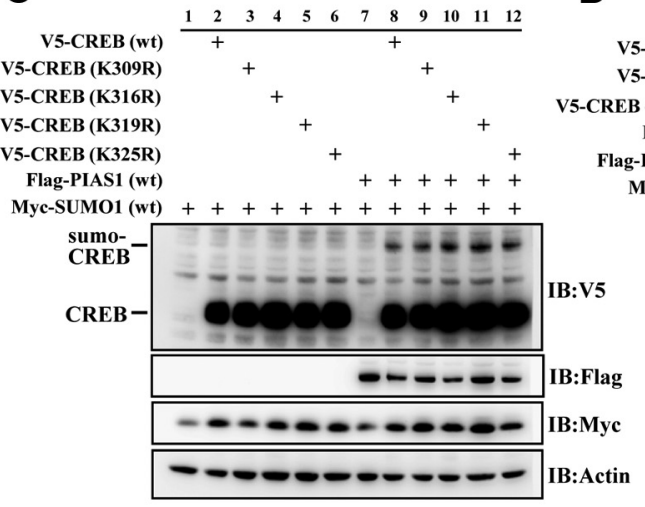

D

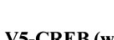

V5-CREB (wt) V5-CREB (K271R) V5-CREB (K290R) $B$ (K271R/K290R) Flag-PIAS1 (wt) lag-PIAS1 (W372A)

Myc-SUMO1 (wt)
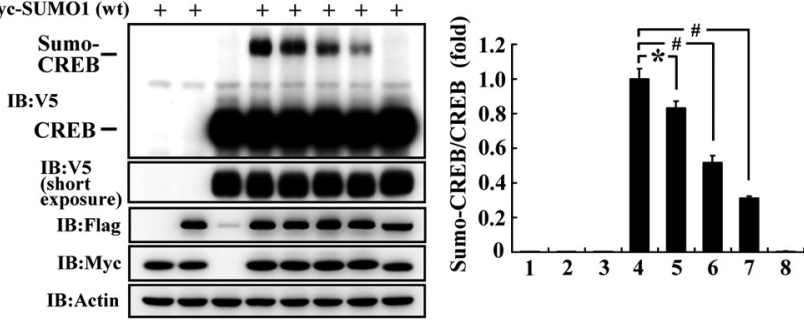

E

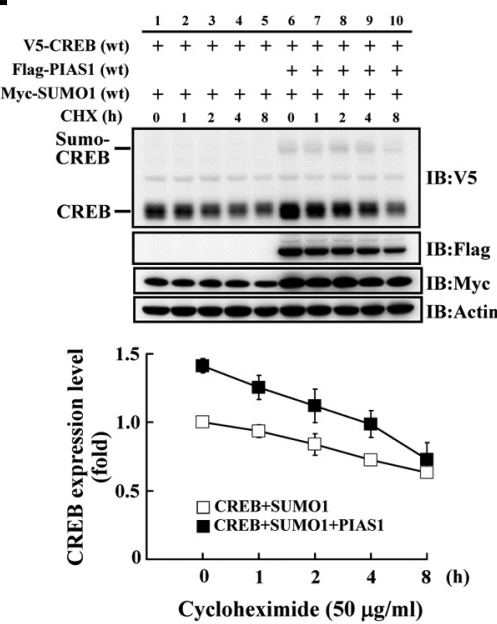

$\mathbf{F}$

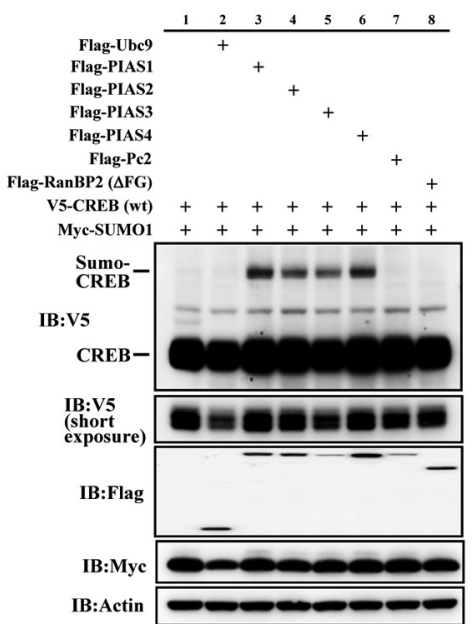

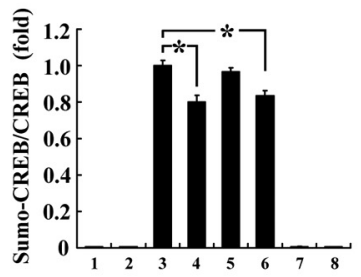

$$
\text { . }
$$

Figure 2. Identification of the major SUM0 acceptors on CREB. A, Examination of PIAS1 SUM0ylation of CREB at Lys109, Lys122, Lys141, Lys271, and Lys278 in HEK293T cells. B, Examination of PIAS1 SUMOylation of CREB at Lys289, Lys290, Lys291, and Lys295 in HEK293T cells. C, Examination of PIAS1 SUMOylation of CREB at Lys309, Lys316, Lys319, and Lys325 in HEK293T cells. D, Confirmation of PIAS1 SUMOylation of CREB at Lys271 and Lys290 in HEK293T cells. The quantified result is shown on the right. $\boldsymbol{E}$, PIAS1 overexpression increased the expression level of CREB in HEK293T cells. The quantified result over time is shown below. CHX, Cycloheximide. Data are shown as mean \pm SEM. F, CREB SUMOylation by PIAS family proteins, other E3 ligases, and E2 ligase in HEK293T cells. The quantified result is shown on the right. $\Delta \mathrm{FG}$, Partial sequence of full-length RanBP2. Data are expressed as mean \pm SEM. ${ }^{*} p<0.05$; \#p $<0.001$. Experiments were performed in duplicate or triplicate.

fected ( $q=5.43, p<0.01$ vs the Flag-vector group; Fig. $3 B$, left, lane 2 vs lane 1), but this effect diminished when FlagCREBK271R or Flag-CREBK290R sumo-mutant was transfected $(q=7.51, p<0.001$ and $q=8.63, p<0.001$, respectively, vs the Flag-CREBWT group; Fig. $3 B$, right). Further, transfection of the Flag-CREB double sumo-mutant completely blocked the effect of PIAS1 on CREB SUMOylation $(q=11.12, p<0.001$; Fig. $3 B$, left, lane 5 vs lane 2). Addition of the SUMO1 mutant protein to the reaction also abolished the effect of PIAS1 on CREB SUMOylation ( $q=11.11, p<0.001$; Fig. $3 B$, left, lane 6 vs lane 2$)$. The quantified result is shown in Figure $3 B$, right. These results were further verified by Western blot against SUMO1. A representative gel pattern of CREB SUMOylation under the same conditions is shown in Figure $3 C$, left. Statistical analyses similarly 
A

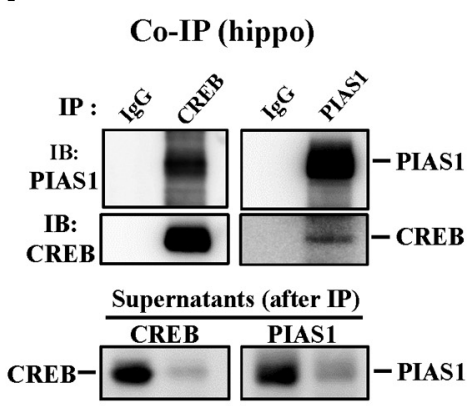

B

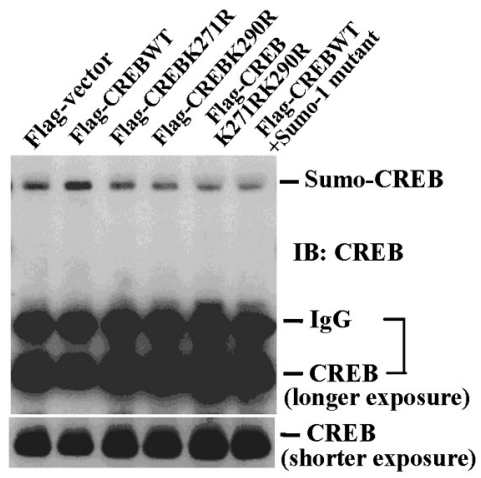

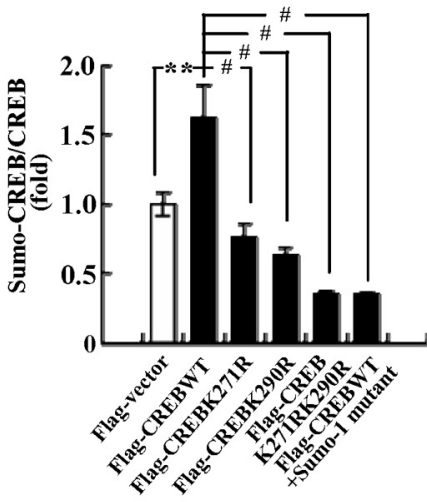

D

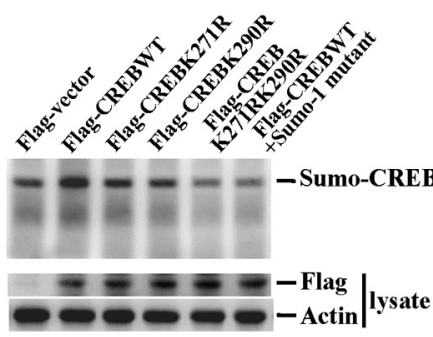

IB: Sumo-1

E

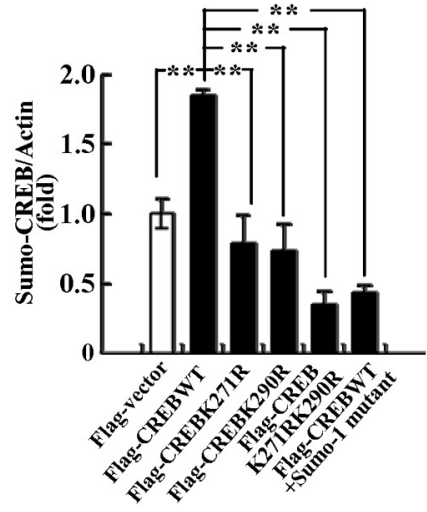

$\mathbf{F}$
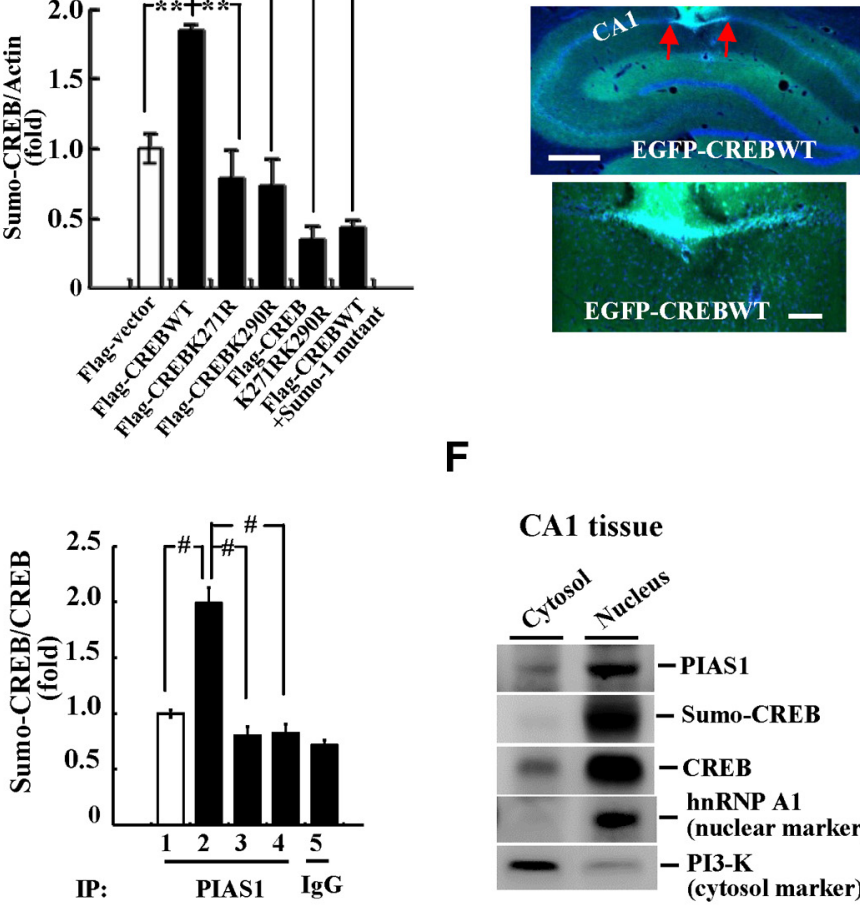

CA1 tissue

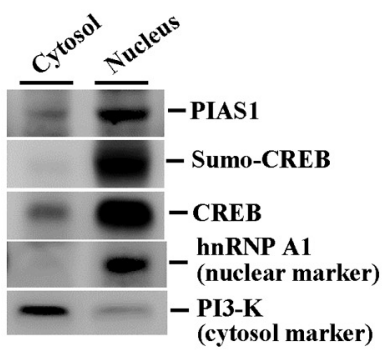

1. Flag-vector+PBS

2. Flag-vector+NMDA

3. Flag-vector+NMDA+

Sumo-1 mutant protein

4. Flag-PIAS1W372A+NMDA

5. Flag-vector+NMDA

Figure 3. CREB is sumoylated by PIAS1 in the hippocampus. $A$, Co-IP showing that PIAS1 is associated with CREB and vice versa in rat hippocampus. Identification of CREB sumo acceptors in rat hippocampus by Western blot against CREB $(\boldsymbol{B})$ and SUM01 ( $\boldsymbol{C}$. The quantified results are shown on the right. $\boldsymbol{D}$, Confocal images showing EGFP-CREB transfection and expression in the CA1 area at different magnifications. Cells that show both green fluorescence (EGFP) and blue fluorescence (DAPI) are cells successfully transfected with the plasmid. Red arrows indicate the area of transfection. Scale bars: top, $500 \mu \mathrm{m}$; bottom, $100 \mu \mathrm{m}$. E, NMDA was injected into rat CA1 area bilaterally (1.2 $\mu \mathrm{g} / \mu \mathrm{l}, 0.7 \mu \mathrm{l}$ each side) and in vitro SUM0ylation for CREB was performed 30 min later. In one group, the SUM01 mutant protein was added to the reaction and, in another group, the ligase-deficient mutant Flag-PIAS1W372A plasmid was transfected. Effect of NMDA injection was confirmed by elevated level of pCREB from Western blot in a separate analysis. Flag-PIAS1W372A transfection was confirmed by Western blot against the Flag tag. $n=5$ each group. The quantified result is shown on the right. $\boldsymbol{F}$, Subcellular distribution of PIAS1, CREB, and the sumoylated form of CREB in the cytosol and nucleus fractions from the rat CA1 tissue. Data are expressed as mean \pm SEM. ${ }^{* *} p<0.01 ; \# p<0.001$. Experiments were performed in duplicate or triplicate.

revealed an overall significant effect of these transfections on CREB SUMOylation $\left(F_{(5,6)}=16.48, p<0.01\right)$. Further analyses indicated that CREB SUMOylation by PIAS1 was increased when the Flag-CREBWT plasmid was transfected $(q=6.35, p<0.01 \mathrm{vs}$ the Flag-vector group; Fig. $3 C$, left, lane 2 vs lane 1), but this effect was reduced when the Flag-CREBK271R or Flag-CREBK290R sumo-mutant was transfected $(q=7.97, p<0.01$ and $q=8.36$, $p<0.01$, respectively, vs the Flag-CREBWT group; Fig. $3 C$, right). Further, transfection of Flag-CREBK271RK290R completely blocked the effect of PIAS1 on CREB SUMOylation $(q=$ $11.25, p<0.01$ ). Addition of the SUMO1 mutant protein also prevented the effect of PIAS1 on CREB SUMOylation $(q=10.64$, $p<0.01$; Fig. $3 C$, left, lane 6 vs lane 2). The quantified results are shown in Figure $3 C$, right. Flag-CREB plasmid transfection and expression was confirmed by Western blot against the Flag tag (Fig. 3C, left) and further confirmed by immunohistochemistry 
showing EGFP fluorescence in the CA1 area upon EGFP-CREBWT plasmid transfection (Fig. 3D).

\section{NMDA injection increases CREB SUMOylation}

NMDA receptor activation and signaling play a key role in mammalian learning and memory formation (Collingridge, 1987; Izquierdo, 1991). In this experiment, we investigated whether NMDA injection produces a similar effect on CREB SUMOylation as spatial training. Animals were divided into 5 groups $(n=5$ each group) to receive Flag-vector (or FlagPIAS1W372A) transfection and NMDA $(1.2 \mu \mathrm{g} / \mu \mathrm{l}$; or PBS) injection to the CA1 area. They were decapitated $30 \mathrm{~min}$ after NMDA injection and their CA1 tissue was dissected out. The cell lysate was immunoprecipitated with PIAS1 antibody (or IgG) and subjected to in vitro SUMOylation assay for PIAS1 SUMOylation of CREB. In one group, the SUMO1 mutant protein was added to the reaction during the SUMOylation assay. Results revealed an overall significant effect of these treatments on CREB SUMOylation $\left(F_{(4,20)}=37.64, p<\right.$ $0.001)$. Further analyses indicated that NMDA injection markedly increased the level of CREB SUMOylation $(q=11.52$, $p<0.001$; Fig. $3 E$, lane 2 vs lane 1 ). This effect was blocked by addition of the SUMO1 mutant protein $(q=13.86, p<$ 0.001 vs the NMDA group; Fig. $3 E$, lane 3 vs lane 2) and by transfection of FlagPIAS1W372A $(q=13.6, p<0.001$ vs the NMDA group; Fig. $3 E$, lane 4 vs lane 2). The effectiveness of NMDA injections was confirmed by the apparent increase in the level of CREB phosphorylation from a separate Western blot (Fig. 3E). FlagPIAS1W372A transfection and expression were confirmed by Western blot against the Flag tag (Fig. 3E, left). The quantified result is shown in Figure $3 E$, right. We next examined the subcellular compartment in which PIAS1 SUMOylation of CREB takes place. The CA1 tissue was fractionated into the cytosol and nucleus fractions and Western blots with different antibodies were performed. The results indicated that both PIAS1 and CREB were mainly distributed in the nucleus and that CREB SUMOylation also takes place in the nucleus (Fig. 3F).

\section{Time course change of CREB} phosphorylation and CREB SUMOylation after spatial training In this experiment, we investigated the time course change of CREB phosphorylation and CREB SUMOylation after spa-
A

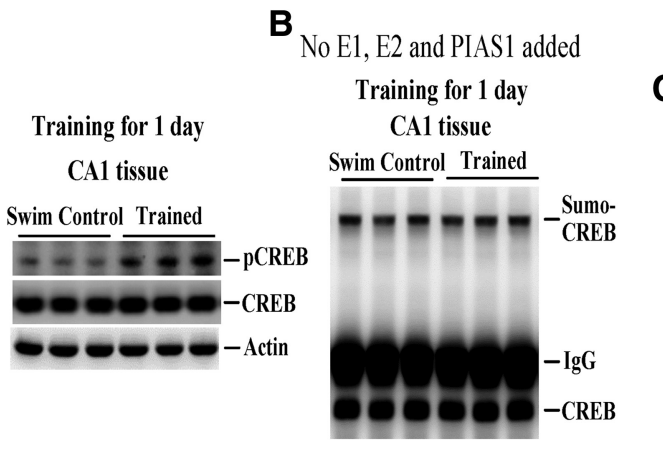

D

E No E1, E2 and PIAS1 added

Training for 2 days

CA1 tissue

Training for 2 days

CA1 tissue

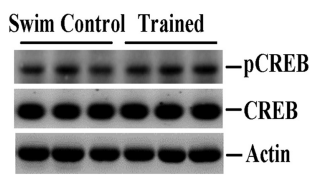

G

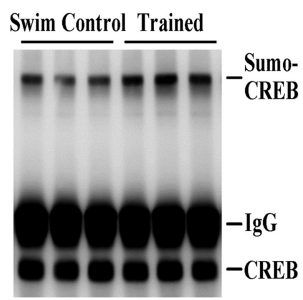

H

No E1, E2 and PIAS1 added

Training for 5 days

Training for 5 days

CA1 tissue

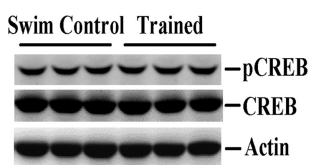

$\mathbf{J}$

\section{C}
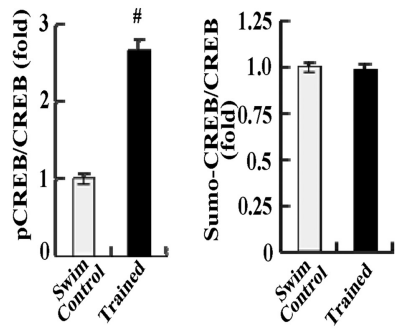

$\mathbf{F}$
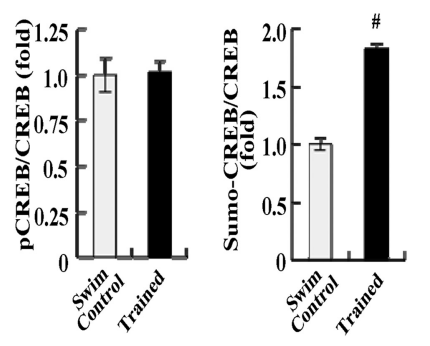

No E1, E2 and PIAS1 added

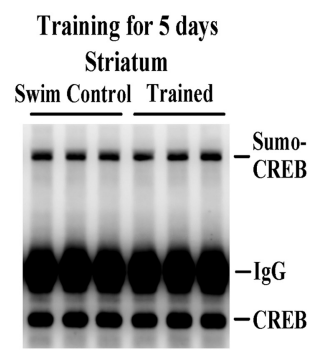

CA1 tissue

Swim Control Trained
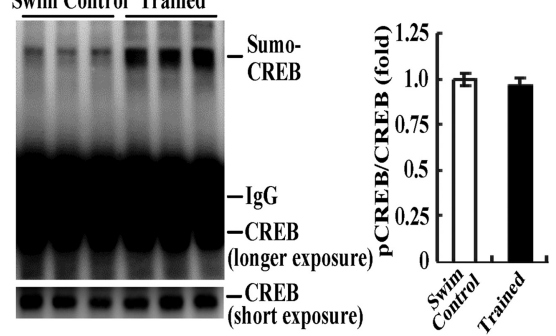

K

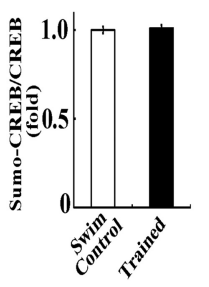

No E1, E2 and PIAS1 added

Training for 5 days Amygdala

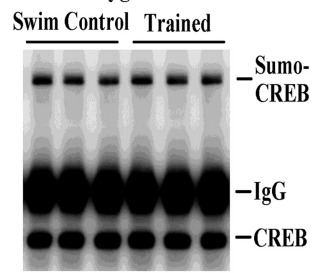

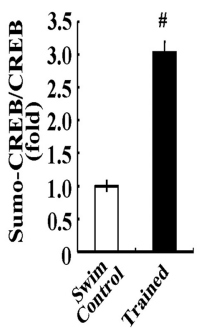

Figure 4. Time course change of CREB phosphorylation and CREB SUMOylation in rat hippocampus. Naive rats were randomly assigned to the swim control group or subjected to spatial training for $1 \mathrm{~d}$ and their CA1 tissue was dissected out for determination of CREB phosphorylation (A) and endogenous CREB SUMOylation $(\boldsymbol{B})$. The quantified results are shown in C. Separate groups of animals were divided into the swim control group or subjected to spatial training for $2 \mathrm{~d}$ and their CA1 tissue was dissected out for determination of CREB phosphorylation (D) and endogenous (REB SUMOylation ( $\boldsymbol{E})$. The quantified results are shown in $\boldsymbol{F}$. Two other groups of animals were divided into the swim control group or subjected to spatial training for $5 \mathrm{~d}$ and their CA1 tissue was dissected out for determination of CREB phosphorylation $(\boldsymbol{G})$ and endogenous (REB SUMOylation $(\boldsymbol{H})$. The quantified results are shown in $\boldsymbol{I}$. Endogenous CREB SUMOylation in the striatum $(\boldsymbol{J})$ and amygdala $(\boldsymbol{K})$ from the same animals (trained for $5 \mathrm{~d}$ ) and the quantified results are shown. $n=5$ each group. Data are expressed as mean \pm SEM. \#p $<0.001$. 
A

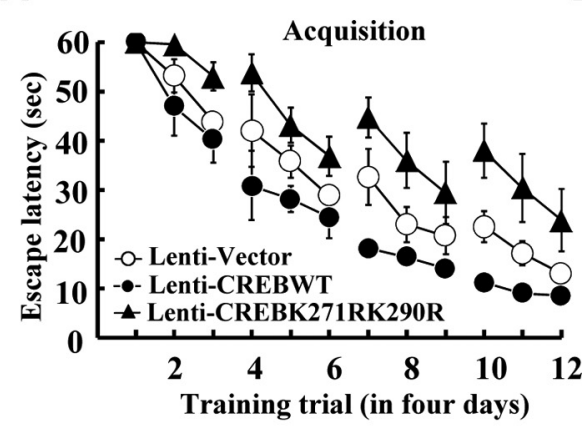

C
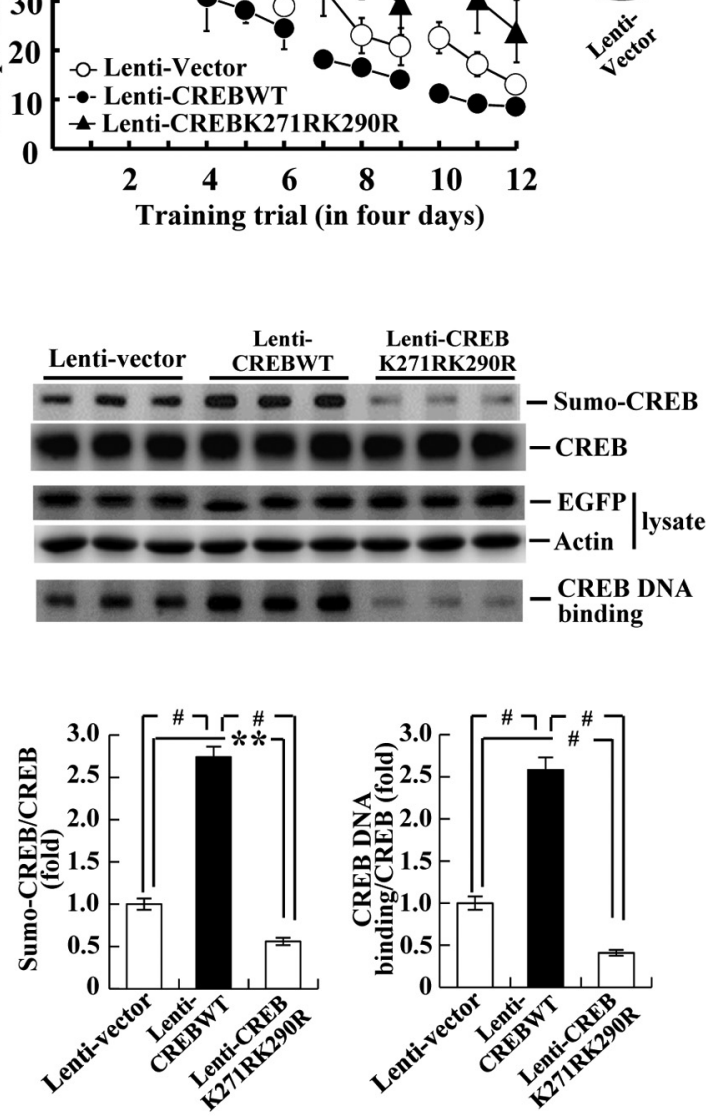
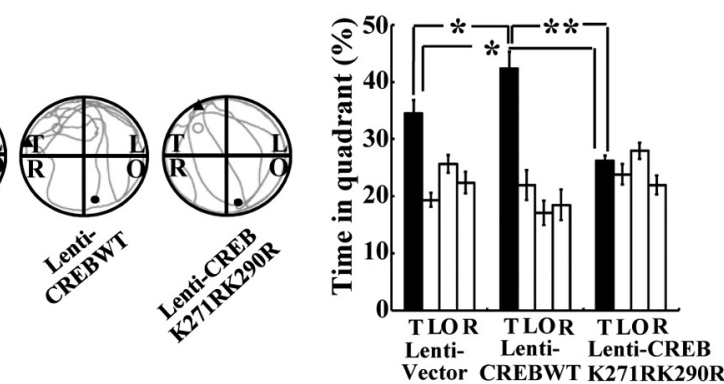

D

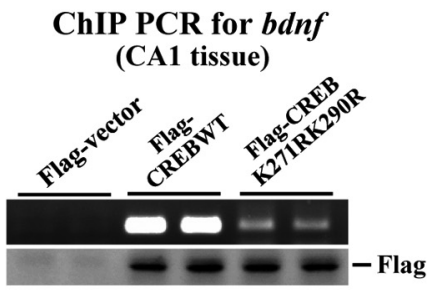

ChIP Q-PCR for bdnf

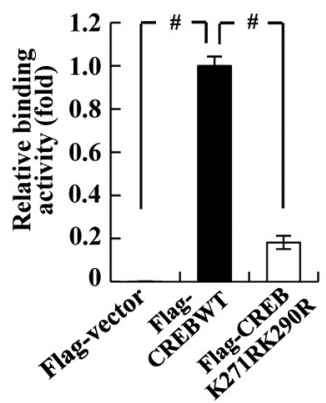

$\mathbf{E}$

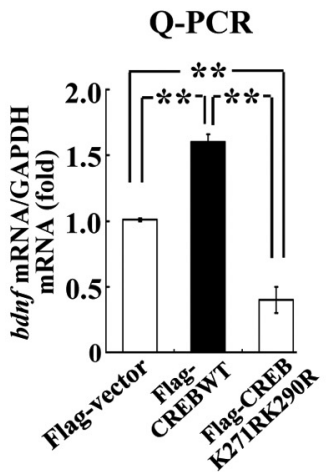

$\mathbf{F}$

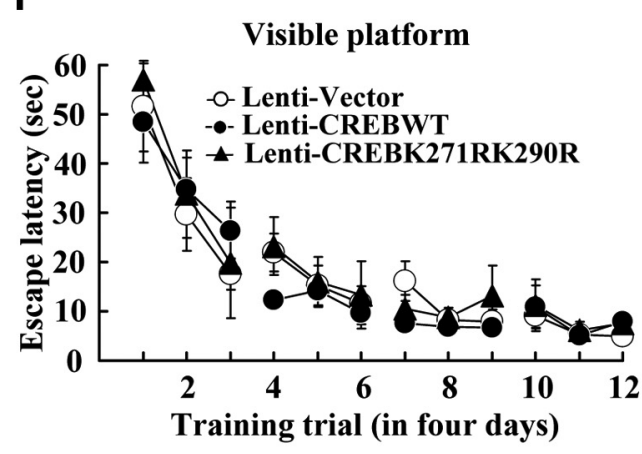

Figure 5. CREB SUMOylation enhances spatial learning and memory and increases CREB DNA binding and bdnf mRNA expression. Effects of Lenti-CREBWT and Lenti-CREB sumo-mutant transduction to rat CA1 area on spatial acquisition $(\boldsymbol{A})$, probe trial performance $(\boldsymbol{B})$, CREB SUMOylation and CREB DNA binding (C). $n=7$ each group. D, ChIP PCR (top) and ChIP qPCR (bottom) showing the effects of Flag-CREBWT and Flag-CREB sumo-mutant transfection on CREB DNA binding to the bdnf promoter. E, Quantitative PCR showing the effects of Flag-CREBWT and Flag-CREB sumo-mutant transfection on bndf mRNA level. $\boldsymbol{F}$, Effect of Lenti-CREBWT and Lenti-CREB sumo-mutant transduction to rat CA1 area on visible platform learning ( $n=4$ each group). Data are expressed as mean \pm SEM. Biochemical experiments were performed in duplicate. ${ }^{*} p<0.05 ;{ }^{* *} p<0.01 ; \# p<0.001$.

tial learning and studied the relationship between these two. Different groups of animals were subjected to spatial training for different periods $(1,2$, and $5 \mathrm{~d}, n=5$ each group). They were decapitated at the end of training and their CA1 tissue was dissected out and subjected to CREB phosphorylation and endogenous CREB SUMOylation determinations. The reason that we chose these time points was to cover a wide range after training so that both the activation (phosphorylation) and inactivation (dephosphorylation) of CREB could be included and CREB SUMOylation could be determined at the same time points. A representative gel pattern of CREB phosphorylation and CREB SUMOylation at $1 \mathrm{~d}$ after training is shown in Figure $4, A$ and $B$, 
A

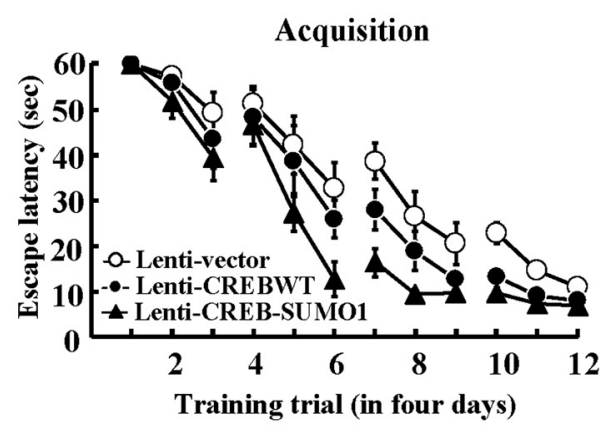

C
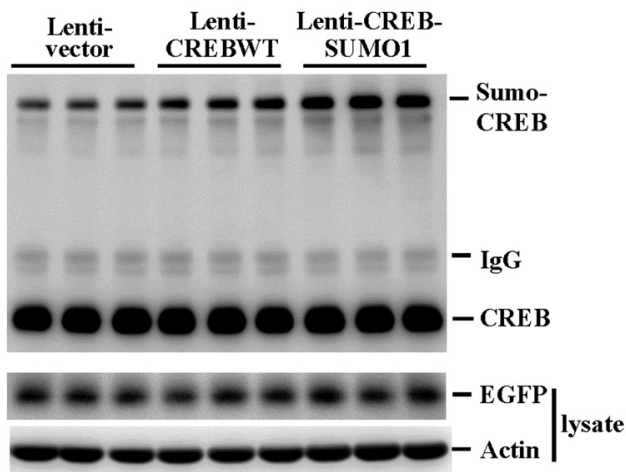

B
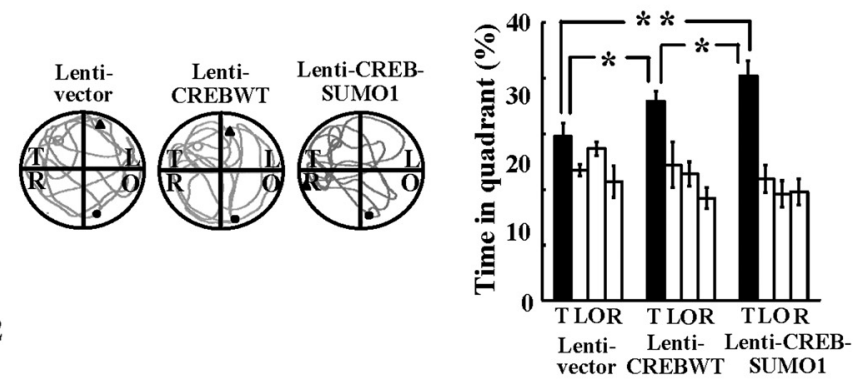

D
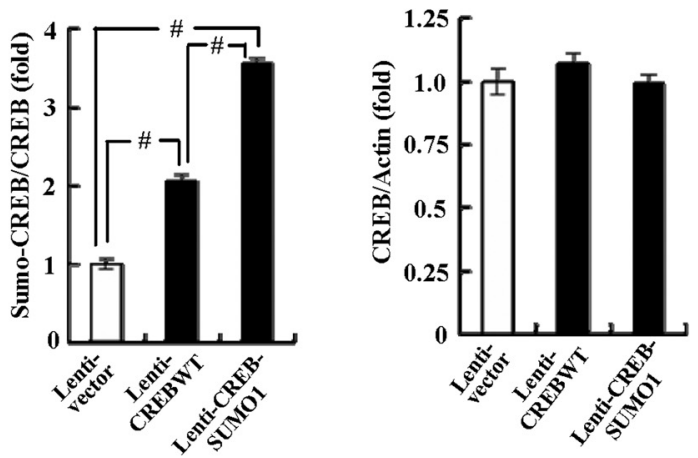

Figure 6. Lenti-CREB-SUM01 fusion vector transduction enhances spatial learning and memory and increases CREB SUMOylation. Effects of Lenti-CREBWT and Lenti-CREB-SUM01 fusion vector transduction to the rat CA1 area on spatial acquisition $(\boldsymbol{A})$, probe trial performance $(\boldsymbol{B})$, and CREB SUMOylation $(\boldsymbol{C})$ are shown. The quantified result of CREB SUMOylation is shown in $\boldsymbol{D}$. $n=8$ each group. Data are expressed as mean \pm SEM. ${ }^{*} p<0.05 ;{ }^{* *} p<0.01 ; \# p<0.001$.

respectively. Statistical analysis revealed that spatial training for $1 \mathrm{~d}$ significantly increased the level of CREB phosphorylation $\left(t_{(1,8)}=11.66, p<0.001\right)$, but the level of CREB SUMOylation was not altered $\left(t_{(1,8)}=0.29, p>0.05\right.$; Fig. $\left.4 C\right)$. A representative gel pattern of CREB phosphorylation and CREB SUMOylation at $2 \mathrm{~d}$ after training is shown in Figure 4, D and $E$, respectively. Statistical analysis revealed that spatial training for $2 \mathrm{~d}$ no longer affected the level of CREB phosphorylation $\left(t_{(1,8)}=0.14, p>\right.$ $0.05)$, but did increase the level of CREB SUMOylation $\left(t_{(1,8)}=\right.$ 12.45, $p<0.001$; Fig. $4 F$ ). A representative gel pattern of CREB phosphorylation and CREB SUMOylation at $5 \mathrm{~d}$ after training is shown in Figure 4, $G$ and $H$, respectively. Statistical analysis revealed that spatial training for $5 \mathrm{~d}$ also did not affect the level of CREB phosphorylation $\left(t_{(1,8)}=0.64, p>0.05\right)$, but consistently and markedly increased the level of CREB SUMOylation $\left(t_{(1,8)}=\right.$ 11.44, $p<0.001$; Fig. 4I). These results together suggest that CREB phosphorylation takes place at an early stage, whereas CREB SUMOylation takes place at a later stage after training.

Next, we investigated whether spatial-training-induced CREB SUMOylation is specific to the hippocampus. Endogenous CREB SUMOylation in the striatum and amygdala from the same animals subjected to spatial training for $5 \mathrm{~d}$ was determined. Results revealed that CREB SUMOylation in the striatum and amygdala was not altered at $5 \mathrm{~d}$ after spatial training $\left(t_{(1,8)}=0.24, p>0.05\right.$ and $t_{(1,8)}=$ $0.19, p>0.05$, respectively; Fig. 4 , J and $K)$. These results suggest that the increase in CREB SUMOylation is learning dependent.

CREB SUMOylation facilitates spatial learning and memory and enhances CREB DNA binding and $\boldsymbol{b} d \boldsymbol{d} f$ gene expression To determine the effect of CREB SUMOylation on water maze performance, we transducted Lenti-CREBWT or Lenti-CREB double sumo-mutant to the rat CA1 area ( $n=7$ each group) and these animals were subjected to water maze learning. Results revealed that Lenti-CREBWT transduction markedly enhanced $\left(F_{(2,18)}=14.18\right.$, $p<0.001 ; q=3.17, p<0.05)$, whereas Lenti-CREB sumo-mutant transduction markedly impaired $(q=4.33, p<0.01)$, acquisition performance in rats (Fig. $5 A$ ). Probe trial analyses indicated that the Lenti-CREBWT-transducted animals spent more time in the target quadrant $\left(F_{(2,18)}=14.94, p<0.001 ; q=3.78, p<0.05\right)$, whereas the Lenti-CREB sumo-mutant transducted animals spent less time in the target quadrant $(q=3.95, p<0.05$; Fig. $5 B$, right). A representative swimming pattern of rats from each group is shown in Figure $5 B$, left. Further biochemical analyses revealed that Lenti-CREBWT transduction significantly increased CREB SUMOylation $\left(F_{(2,18)}=\right.$ 182.94, $p<0.001 ; q=20.42, p<0.01)$ and CREB DNA binding $\left(F_{(2,18)}=129.78, p<0.001 ; q=16.05, p<0.001\right)$, whereas LentiCREB sumo-mutant transduction markedly decreased CREB SUMOylation $(q=5.16, p<0.01)$ and CREB DNA binding $(q=5.98$, $p<0.001$; Fig. $5 C$ ). Lentiviral vector transduction and protein expression were confirmed by Western blot against EGFP (Fig. 5C).

Because CREB regulates the expression of $b d n f$ (Tao et al., $1998)$ and $b d n f$ plays an important role in learning and memory formation (Ma et al., 1998; Mizuno et al., 2000), we further investigated whether CREB SUMOylation enhances CREB binding to the $b d n f$ promoter and $b d n f$ gene expression. Results from ChIP PCR showed the gel pattern of Flag-CREBWT and FlagCREBK271RK290R binding to the $b d n f$ promoter in rat CA1 area (Fig. 5D, top). Further results from ChIP Q-PCR revealed that Flag-CREBWT transfection markedly enhanced CREB DNA binding to the $b d n f$ promoter $\left(F_{(2,11)}=256.8, p<0.001 ; q=\right.$ 29.22, $p<0.001$ ), but this effect was significantly blocked by Flag-CREB sumo-mutant transfection $(q=25.39, p<0.001$; Fig. 
A

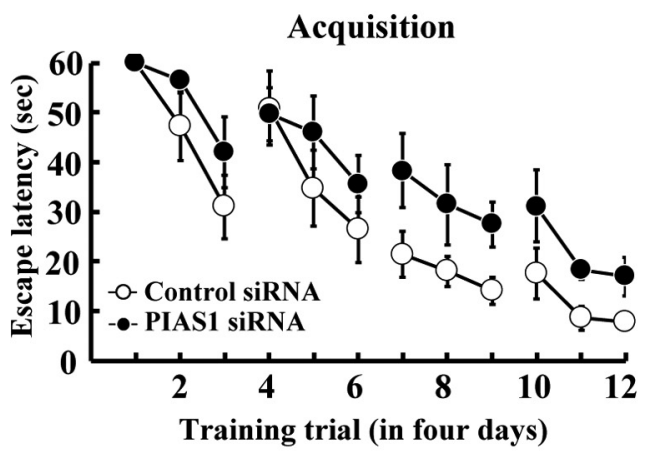

C

No E1, E2 and PIAS1 added
B
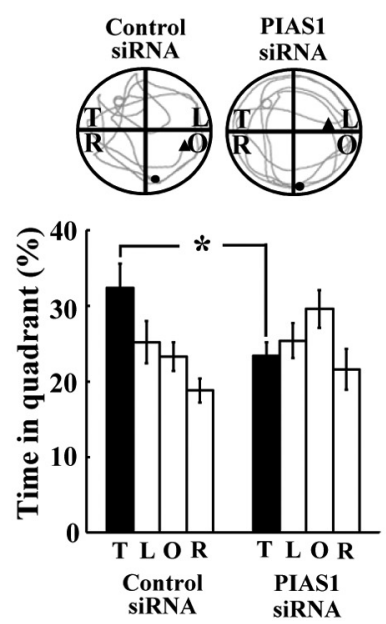

CA1 tissue

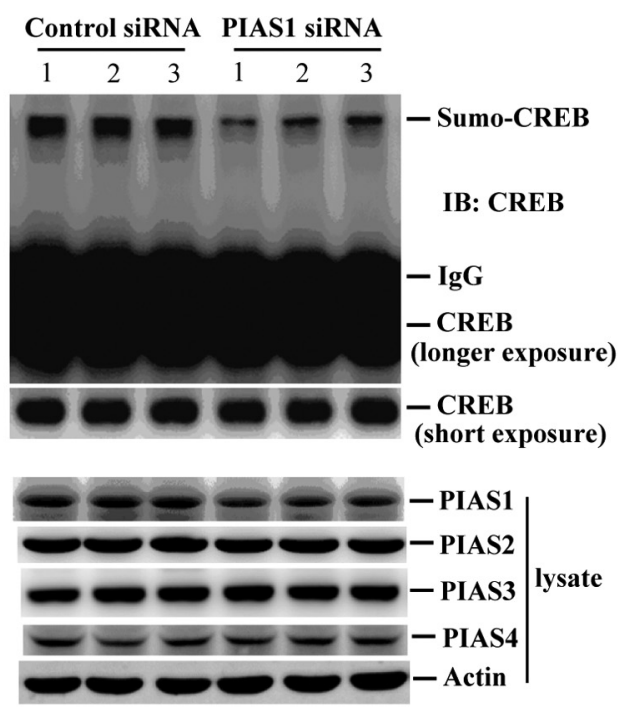

D
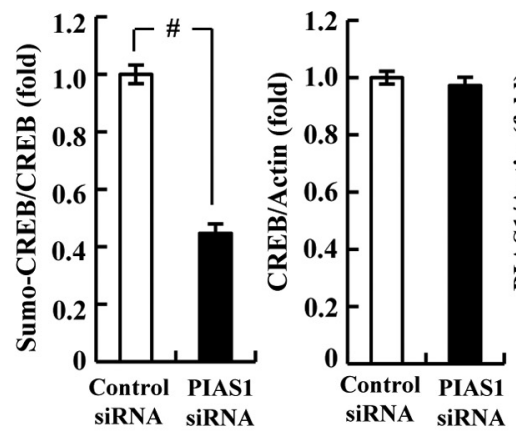

Figure 7. PIAS1 siRNA transfection impairs water maze performance and decreases (REB SUMOylation. Effects of PIAS1 siRNA transfection to the rat CA1 area on spatial acquisition ( $A$ ), probe trial performance $(\boldsymbol{B})$, endogenous CREB SUMOylation and the expression of different PIAS family proteins $(\boldsymbol{C})$ are shown. The quantified result of CREB SUMOylation is shown in $\boldsymbol{D}$. $n=7$ each group. Data are expressed as mean \pm SEM. ${ }^{*} p<0.05 ; \# p<0.001$.

$5 D$, bottom). In addition, Flag-CREBWT transfection increased $\left(F_{(2,3)}=83.36, p<0.01 ; q=8.96, p<0.01\right)$, whereas Flag-CREB sumo-mutant transfection decreased $(q=9.3, p<0.01)$, the $b d n f$ mRNA level (Fig. $5 E$ ). In a separate experiment, we investigated the effect of Lenti-CREBWT transduction and Lenti-CREB sumomutant transduction to CA1 area on visible platform learning in rats and the results revealed that their visible platform learning performance was not different $\left(F_{(2,14)}=0.33, p>0.05\right.$; Fig. $\left.5 F\right)$.

CREB-SUMO1 fusion vector transduction enhances spatial learning and memory and increases CREB SUMOylation To further examine the role of CREB SUMOylation in spatial learning and memory, we transducted Lenti-CREBWT or LentiCREB-SUMO1-fusioned vector to rat CA1 area $(n=8$ each group) and these animals were subjected to water maze learning. Results revealed that Lenti-CREBWT transduction facilitated acquisition performance in rats $\left(F_{(2,21)}=10.47, p<0.001 ; q=\right.$ $3.23, p<0.05)$, and this effect was further enhanced when LentiCREB-SUMO1 was transducted $(q=3.24, p<0.05$ vs the
CREBWT group; Fig. 6A). Probe trial analyses indicated that the Lenti-CREBWT-transducted animals spent more time in the target quadrant $\left(F_{(2,21)}=9.79, p<0.001 ; q=3.06, p<0.05\right)$ and this was more evident upon Lenti-CREB-SUMO1 transduction $(q=3.19$, $p<0.05$; Fig. $6 B$, right). A representative swimming pattern of rat from each group is shown in Figure $6 B$, left. Further biochemical analyses revealed that Lenti-CREBWT transduction significantly increased CREB SUMOylation $\left(F_{(2,21)}=247.98, p<0.001 ; q=12.89\right.$, $p<0.001)$ and this effect was further enhanced upon Lenti-CREBSUMO1 transduction ( $q=18.44, p<0.001$ vs the CREBWT group; Fig. $6 C, D)$. Lentiviral vector transduction and protein expression were confirmed by Western blot against EGFP (Fig. 6C).

PIAS1 siRNA transfection impairs spatial learning and memory and decreases CREB SUMOylation

The above experiments demonstrated the role of endogenous CREB SUMOylation by PIAS1 involved in spatial learning and memory formation. To further examine the relationship among them, we adopted the PIAS1 siRNA transfection strategy. Ani- 
A

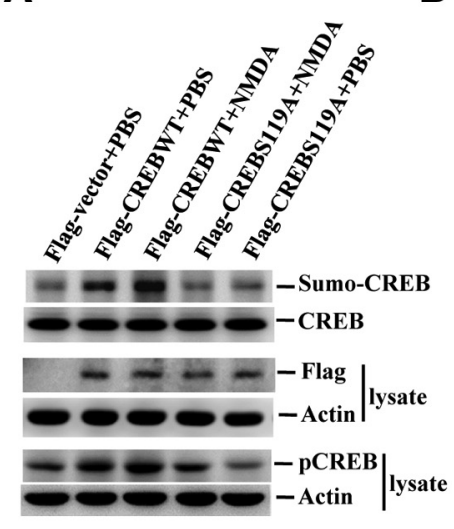

B

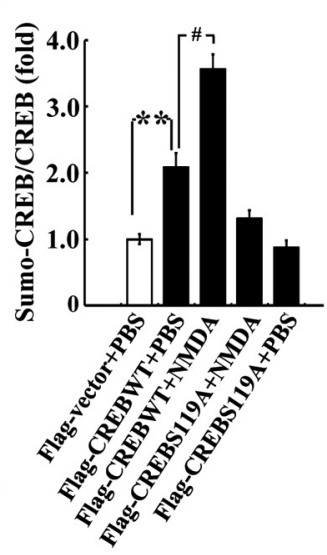

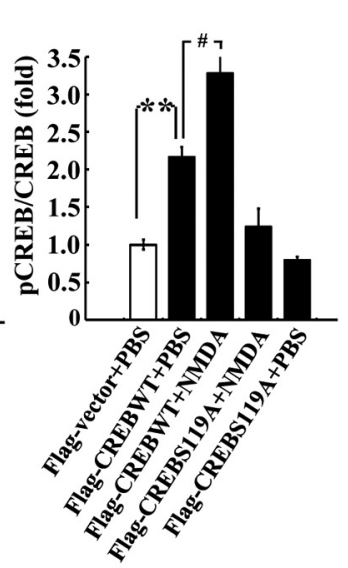

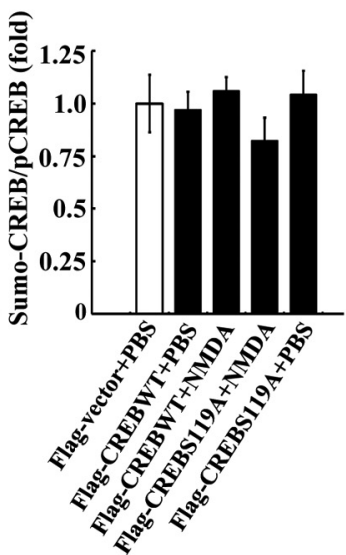

C
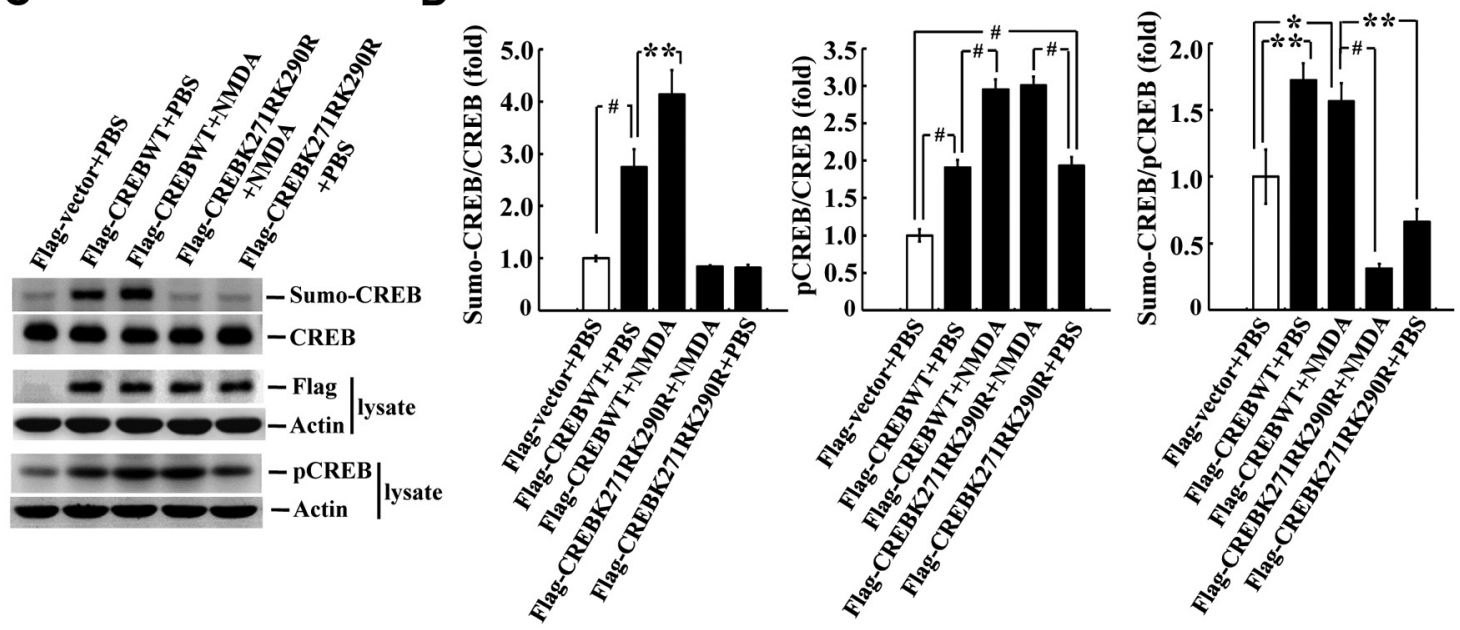

Figure 8. CREB SUMOylation is CREB phosphorylation dependent. $A$, Flag-CREBWT or Flag-CREBS119A plasmid was transfected to rat CA1 area, with or without NMDA coinjection, and CREB SUM0ylation and CREB phosphorylation were determined by Western blot. The quantified results are shown in B. C, Flag-CREBWT or Flag-CREBK271RK290R was transfected to rat CA1 area, with or without NMDA coinjection, and CREB SUMOylation and CREB phosphorylation were determined by Western blot. The quantified results are shown in $\boldsymbol{D} . n=3$ each group. Data are shown as mean \pm SEM. ${ }^{*} p<0.05 ;{ }^{* *} p<0.01 ; \# p<0.001$.

mals were randomly divided into two groups to receive PIAS1 siRNA or control siRNA transfection to the CA1 area and subjected to water maze learning ( $n=7$ each group). Their CA1 tissue was dissected out for endogenous CREB SUMOylation determination. Results revealed that PIAS1 siRNA transfection markedly impaired acquisition performance in rats $\left(F_{(1,12)}=\right.$ $8.45, p=0.01$; Fig. $7 A$ ). Probe trial analysis indicated that animals receiving PIAS1 siRNA transfection spent less time in the target quadrant $\left(t_{(1,12)}=2.60, p<0.05\right.$; Fig. $\left.7 B\right)$. Further biochemical analyses revealed that PIAS1 siRNA transfection significantly decreased the level of endogenous CREB SUMOylation in the CA1 area $\left(t_{(1,12)}=11.98, p<0.001\right.$; Fig. $\left.7 C, D\right)$. The effectiveness of PIAS1 siRNA transfection was confirmed by a marked reduction of PIAS1 expression in the CA1 area $\left(t_{(1,12)}=8.71, p<0.001\right)$. However, the same PIAS1 siRNA transfection did not affect the expression level of PIAS2, PIAS3, and PIAS4 (all $p>0.05$; Fig. $7 C, D)$.

CREB SUMOylation is CREB phosphorylation dependent CREB phosphorylation plays a critical role in mediating longterm memory formation, so we next investigated whether CREB SUMOylation is CREB phosphorylation dependent and vice versa. Flag-CREBWT plasmid or the Flag-CREB phosphoryla- tion mutant plasmid CREBS119A was transfected to rat CA1 area alone or in combination with NMDA ( $n=3$ each group) and CREB SUMOylation and CREB phosphorylation were determined. For the CREB SUMOylation assay, exogenous E1, E2, and the recombinant PIAS1 protein were added to the reaction. A representative gel pattern of CREB SUMOylation is shown in Figure $8 A$. CREB phosphorylation from a separate Western blot is also shown (Fig. 8A). Further analyses revealed that FlagCREBWT plasmid transfection increased the level of CREB SUMOylation $\left(F_{(4,10)}=49.13, p<0.001 ; q=6.86, p<0.01\right.$ vs the control group) and this effect was further enhanced by NMDA injection $(1.2 \mu \mathrm{g} / \mu \mathrm{l} ; q=9.33, p<0.001$ vs the FlagCREBWT group). However, CREB SUMOylation was completely blocked by transfection of Flag-CREBS119A $(q=0.79$, $p>0.05$ vs the control group; Fig. $8 B$ ). Flag-CREBWT transfection also increased CREB phosphorylation $\left(F_{(4,10)}=34.54, p<\right.$ $0.001 ; q=6.65, p<0.01)$ and this effect was similarly enhanced by NMDA injection $(q=6.36, p<0.001)$. However, CREB phosphorylation was completely blocked by transfection of FlagCREBS119A $(q=1.19, p>0.05$; Fig. $8 B)$. The ratio of sumoCREB to pCREB is also shown; no significant difference between any two groups compared can be seen $\left(F_{(4,10)}=0.80, p>0.05\right.$; Fig. $8 B$ ). These results suggest that overexpression of CREB, with 
or without the administration of NMDA, increased CREB phosphorylation and CREB SUMOylation to a similar extent and that overexpression of CREBS119A, with or without the administration of NMDA, prevented CREB phosphorylation and CREB SUMOylation to a similar extent. Plasmid transfection and expression were confirmed by Western blot against the Flag tag (Fig. 8A). A representative gel pattern of CREB SUMOylation from another experiment and CREB phosphorylation from a separate Western blot are shown in Figure $8 C$. Statistical analyses revealed that Flag-CREBWT transfection markedly increased the level of CREB SUMOylation $\left(F_{(4,10)}=32.76, p<0.001 ; q=6.73\right.$, $p<0.001$ vs the control group) and this effect was similarly enhanced by NMDA injection $(q=5.37, p<0.01$ vs the FlagCREBWT group). However, this increased CREB SUMOylation was completely blocked by Flag-CREBK271RK290R transfection $(q=0.69, p>0.05$ vs the control group; Fig. $8 D)$. Conversely, Flag-CREBWT transfection similarly increased CREB phosphorylation $\left(F_{(4,10)}=56.8, p<0.001 ; q=8.19, p<0.001\right)$ and this effect was further enhanced by NMDA injection $(q=9.39, p<$ 0.001). However, transfection of Flag-CREBK271RK290R increased CREB phosphorylation, just as transfection of the FlagCREBWT plasmid did ( $q=8.36, p<0.001$ vs control), and this effect was also enhanced by NMDA injection $(q=9.69, p<$ 0.001 ; Fig. $8 D$ ). The ratio of sumo-CREB to pCREB is also shown and there is an overall significant effect of these treatments on this measure $\left(F_{(4,10)}=20.27, p<0.001\right)$. Further analyses revealed that overexpression of CREB, with or without the administration of NMDA, both resulted in a more significant effect in enhancing CREB SUMOylation than CREB phosphorylation $(q=5.47, p<$ 0.01 and $q=4.27, p<0.05$, respectively). However, overexpression of CREBK271RK290R, with or without the administration of NMDA, both significantly blocked this effect $(q=9.48, p<$ 0.001 and $q=6.83, p<0.01$ vs the CREBWT + NMDA group; Fig. 8D). The difference between the CREBK271RK290R + NMDA group and the CREBK271RK290R + PBS group was not significant $(q=2.66, p>0.05)$. Plasmid transfection and expression was confirmed by anti-Flag Western blot (Fig. 8C).

\section{Discussion}

We demonstrated previously that PIAS1 facilitates spatial learning and memory performance in rats (Tai et al., 2011). In a study of the molecular mechanisms underlying PIAS1-mediated memory facilitation, we found that PIAS1 SUMOylation of CREB plays a very important role, because transduction of the CREB sumo-mutant vector impaired spatial memory formation, but transduction of the CREB-SUMO1 fusion vector enhanced spatial memory formation. This suggestion is further supported by the result that NMDA injection produced a similar effect on CREB SUMOylation as spatial training and this effect was prevented by Flag-PIAS1W372A transfection. The role of CREB SUMOylation involved in spatial memory formation is also supported by the observation that water maze training increased endogenous CREB SUMOylation in the CA1 area. This result suggests that the endogenous E2 and E3 ligase activity in CA1 neurons is sufficient to enhance CREB SUMOylation after water maze learning. Moreover, the same water maze training did not alter the level of CREB SUMOylation in the striatum and amygdala, suggesting that CREB SUMOylation is learning dependent. However, because we have also found that CREB was not sumoylated by the E2 ligase Ubc9 or by two other E3 ligases, Pc2 and RanBP2, and that, among the PIAS family proteins, only the pias 1 gene was found differentially expressed between the fast learners and the slow learners (Tai et al., 2011), these results

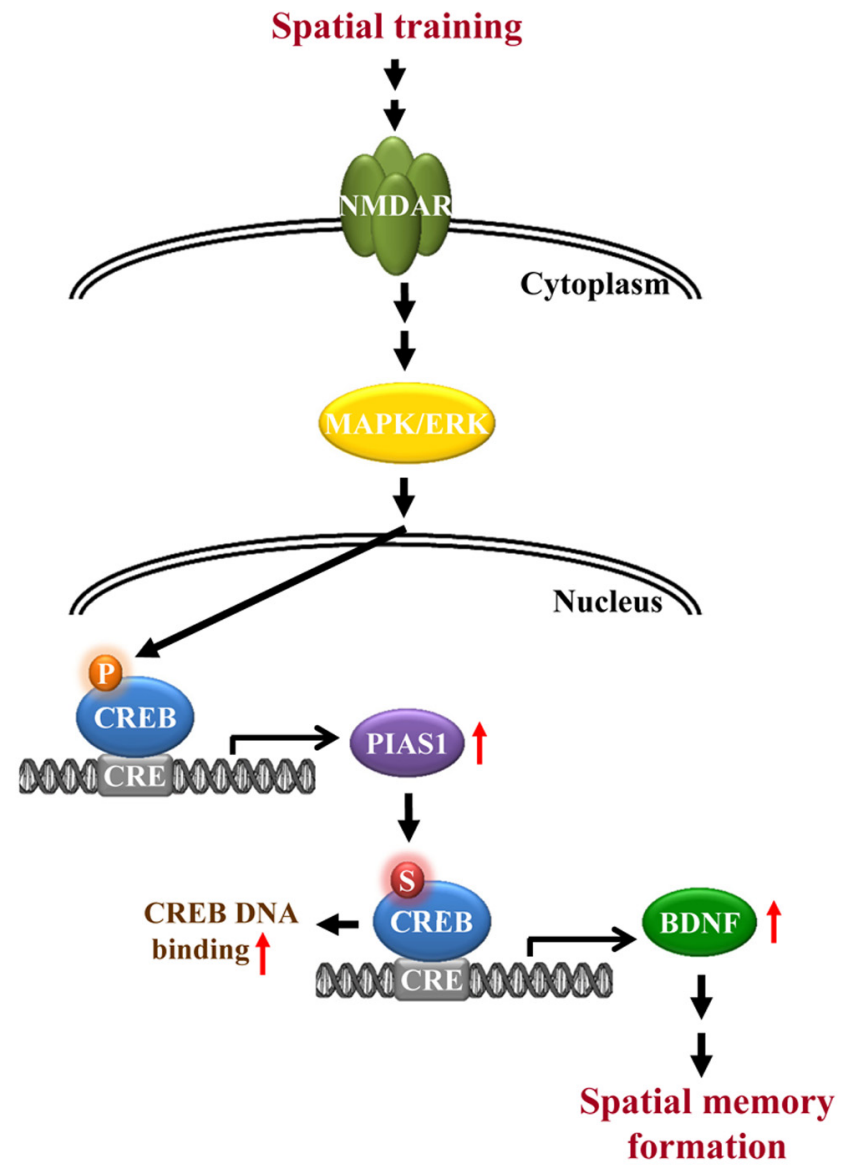

P: phosphorylation S : sumoylation

Figure 9. Schematic diagram showing the relationships among spatial training, PIAS1 expression, CREB SUMOylation, BDNF expression, and spatial memory formation. Spatial training increases PIAS1 expression through activation of the MAPK/ERK signaling cascade (Liu et al., 2013). Increased PIAS1 expression enhances CREB SUMOylation and CREB DNA binding to the gene promoter that upregulates BDNF expression. Increased BDNF expression facilitates spatial memory formation.

together suggest that PIAS1 is perhaps the major E3 ligase that enhances CREB SUMOylation involved in spatial memory formation. This speculation is further supported by our finding that PIAS1 siRNA transfection impaired water maze performance and decreased endogenous CREB SUMOylation.

CREB phosphorylation plays a critical role in long-term memory formation. In our investigation of the relationship between CREB phosphorylation and CREB SUMOylation, we found that CREB phosphorylation takes place earlier than CREB SUMOylation after water maze training and that CREB SUMOylation is CREB phosphorylation dependent. These results are consistent with our previous finding that NMDA receptor activation induces CREB phosphorylation and signaling, which results in increased PIAS1 expression (Liu et al., 2013). Increased PIAS1 expression further enhances CREB SUMOylation. Therefore, increased CREB phosphorylation may be an early event that activates the gene expression necessary for long-term memory formation, whereas CREB SUMOylation sustains long-term memory. This speculation is supported by our finding that a threefold increase in CREB SUMOylation was still observed $5 \mathrm{~d}$ after training, but increased CREB phosphorylation was no longer observed $2 \mathrm{~d}$ after training. Moreover, increased CREB SUMOylation may further enhance 
CREB DNA binding to target genes responsible for long-term memory formation. This speculation is supported by our findings that transfection of the CREB sumo-mutant plasmid decreased CREB binding to the $b d n f$ promoter and decreased $b d n f$ mRNA expression. It is also consistent with another report showing that mutation of CREB Lys304, which corresponds to CREB Lys290 in the present study, blocks CREB interaction with $\mathrm{Mg}^{2+}$ and blocks CREB DNA binding (Craig et al., 2001). It is possible that CREB SUMOylation also enhances CREB binding to other genes associated with memory formation in addition to $b d n f$, a possibility that requires further investigation.

In the present study, we transducted Lenti-CREBWT, LentiCREBK271RK290R, and Lenti-CREB-SUMO1 vectors to rat CA1 area for the water maze experiments because the protein expression lasts longer. For the purposes of transient transfection, Flag-CREBWT and Flag-CREB mutant plasmids were adopted. In addition, the short isoform of the CREB protein $(\mathrm{CREB} 1 \Delta, 327 \mathrm{aa})$ was examined in the present study because CREB $1 \Delta$ is the predominant CREB isoform found in the brain (Blendy et al., 1996) and is also the major clone we obtained from the rat hippocampus. Most of the studies on CREB phosphorylation have focused on Ser133 probably because CREB1 $\alpha$ (the long isoform) was first reported in the literature. In this study, we identified K271 and K290 as the major CREB SUMO acceptors. These two lysine residues correspond to K285 and K304, respectively, for the long isoform of the CREB protein (CREB $1 \alpha, 341$ aa; Mayr and Montminy, 2001), and our results are consistent with a previous study showing that CREB could be sumo-modified by hypoxia at K285 and K304 in T84 cells (Comerford et al., 2003). Likewise, CREB Ser 119 in the present study corresponds to CREB Ser133 in many other studies for CREB phosphorylation.

In addition, for the CREB SUMOylation observed in HEK293T cells, an additional band for endogenous CREB was observed just below the CREB band after IP of V5 (Fig. 1E). We suspect that this band is perhaps due to the CREB dimer formed from the association between transfected V5-CREB and endogenous CREB. Because CREB is dimerized only when it is bound to DNA (Wu et al., 1998), this band may indicate the DNA-bound CREB dimers. Furthermore, our in vivo results revealed that CREB sumo-mutant decreased the level of CREB DNA binding (Fig. 5C), whereas we did not observe an increased expression of the assumed DNA-bound CREB when CREB was sumoylated (Fig. 1E, lane 5). This is probably because the sumoylated CREB observed in lane 5 is mainly contributed by overexpressed V5CREB, not by endogenous CREB; therefore, increased DNAbound endogenous CREB is not readily detected.

CREB phosphorylation and signaling play a critical role in long-term memory formation and CREB glycosylation has been found to impair memory formation. However, other posttranslational modifications of CREB involved in memory formation were not known. Here, we provide the first evidence that CREB could be SUMO-modified by the E3 ligase PIAS1 and that CREB SUMOylation plays an important role in spatial memory formation (Fig. 9). These results are also consistent with the notion that SUMOylation is implicated in synaptic transmission and neuronal plasticity (Scheschonka et al., 2007).

\section{References}

Abdallah B, Hassan A, Benoist C, Goula D, Behr JP, Demeneix BA (1996) A powerful nonviral vector for in vivo gene transfer into the adult mammalian brain: polyethylenimine. Hum Gene Ther 7:1947-1954. CrossRef Medline

Blendy JA, Kaestner KH, Schmid W, Gass P, Schutz G (1996) Targeting of the CREB gene leads to up-regulation of a novel CREB mRNA isoform. EMBO J 15:1098-1106. Medline

Bourtchuladze R, Frenguelli B, Blendy J, Cioffi D, Schutz G, Silva AJ (1994) Deficient long-term memory in mice with a targeted mutation of the cAMP-responsive element-binding protein. Cell 79:59-68. CrossRef Medline

Chao CC, Ma YL, Lee EH (2011) Brain-derived neurotrophic factor enhances Bcl-xL expression through protein kinase casein kinase 2-activated and nuclear factor kappa B-mediated pathway in rat hippocampus. Brain Pathol 21:150-162. CrossRef Medline

Collingridge G (1987) Synaptic plasticity: The role of NMDA receptors in learning and memory. Nature 330:604-605. CrossRef Medline

Comerford KM, Leonard MO, Karhausen J, Carey R, Colgan SP, Taylor CT (2003) Small ubiquitin-related modifier-1 modification mediates resolution of CREB-dependent responses to hypoxia. Proc Natl Acad Sci U S A 100:986-991. CrossRef Medline

Craig JC, Schumacher MA, Mansoor SE, Farrens DL, Brennan RG, Goodman RH (2001) Consensus and variant cAMP-regulated enhancers have distinct CREB-binding properties. J Biol Chem 276:11719-11728. CrossRef Medline

Fleischmann A, Hvalby O, Jensen V, Strekalova T, Zacher C, Layer LE, Kvello A, Reschke M, Spanagel R, Sprengel R, Wagner EF, Gass P (2003) Impaired long-term memory and NR2A-type NMDA receptor-dependent synaptic plasticity in mice lacking c-Fos in the CNS. J Neurosci 23:91169122. Medline

Ihara M, Yamamoto H, Kikuchi A (2005) SUMO-1 modification of PIASy, an E3 ligase, is necessary for PIASy-dependent activation of Tcf-4. Mol Cell Biol 25:3506-3518. CrossRef Medline

Izquierdo I (1991) Role of NMDA receptors in memory. Trends Pharmacol Sci 12:128-129. CrossRef Medline

Jones MW, Errington ML, French PJ, Fine A, Bliss TV, Garel S, Charnay P, Bozon B, Laroche S, Davis S (2001) A requirement for the immediate early gene Zif268 in the expression of late LTP and long-term memories. Nat Neurosci 4:289-296. CrossRef Medline

Kahyo T, Nishida T, Yasuda H (2001) Involvement of PIAS1 in the SUMOylation of tumor suppressor p53. Mol Cell 8:713-718. CrossRef Medline

Liao J, Fu Y, Shuai K (2000) Distinct roles of the NH2- and COOH-terminal domains of the protein inhibitor of activated signal transducer and activator of transcription (STAT) 1 (PIAS1) in cytokine-induced PIAS1-Stat1 interaction. Proc Natl Acad Sci U S A 97:5267-5272. CrossRef Medline

Liu B, Liao J, Rao X, Kushner SA, Chung CD, Chang DD, Shuai K (1998) Inhibition of Stat1-mediated gene activation by PIAS1. Proc Natl Acad Sci U S A 95:10626-10631. CrossRef Medline

Liu B, Mink S, Wong KA, Stein N, Getman C, Dempsey PW, Wu H, Shuai K (2004) PIAS1 selectively inhibits interferon-inducible genes and is important in innate immunity. Nat Immunol 5:891-898. CrossRef Medline

Liu SY, Ma YL, Lee EH (2013) NMDA receptor signaling mediates the expression of protein inhibitor of activated STAT1 (PIAS1) in rat hippocampus. Neuropharmacology 65:101-113. CrossRef Medline

Livak KJ, Schmittgen TD (2001) Analysis of relative gene expression data using real-time quantitative PCR and the 2(-Delta Delta C(T)) method. Methods 25:402-408. CrossRef Medline

Lubin FD, Roth TL, Sweatt JD (2008) Epigenetic regulation of BDNF gene transcription in the consolidation of fear memory. J Neurosci 28:1057610586. CrossRef Medline

Ma YL, Wang HL, Wu HC, Wei JL, Lee EH (1998) BDNF antisense oligonucleotide impairs memory retention and inhibits long-term potentiation in rats. Neuroscience 82:957-967. CrossRef Medline

Mayr B, Montminy M (2001) Transcriptional regulation by the phosphorylation-dependent factor CREB. Nat Rev Mol Cell Biol 2:599_ 609. CrossRef Medline

Mizuno M, Yamada K, Olariu A, Nawa H, Nabeshima T (2000) Involvement of brain-derived neurotrophic factor in spatial memory formation and maintenance in a radial arm maze test in rats. J Neurosci 20:71167121. Medline

Pichler A, Gast A, Seeler JS, Dejean A, Melchior F (2002) The nucleoporin RanBP2 has SUMO1 E3 ligase activity. Cell 108:109-120. CrossRef Medline

Rexach JE, Clark PM, Mason DE, Neve RL, Peters EC, Hsieh-Wilson LC (2012) Dynamic O-GlcNAc modification regulates CREB-mediated gene expression and memory formation. Nat Chem Biol 8:253-261. CrossRef Medline 
Rytinki MM, Kaikkonen S, Pehkonen P, Jääskeläinen T, Palvimo JJ (2009) PIAS proteins: pleiotropic interactors associated with SUMO. Cell Mol Life Sci 66:3029-3041. CrossRef Medline

Scheschonka A, Tang Z, Betz H (2007) SUMOylation in neurons: nuclear and synaptic roles? Trends Neurosci 30:85-91. CrossRef Medline

Shuai K, Liu B (2003) Regulation of JAK-STAT signalling in the immune system. Nat Rev Immunol 3:900-911. CrossRef Medline

Silva AJ, Kogan JH, Frankland PW, Kida S (1998) CREB and memory. Annu Rev Neurosci 21:127-148. CrossRef Medline

Tai DJ, Hsu WL, Liu YC, Ma YL, Lee EH (2011) Novel role and mechanism of protein inhibitor of activated STAT1 in spatial learning. EMBO J 30: 205-220. CrossRef Medline

Tao X, Finkbeiner S, Arnold DB, Shaywitz AJ, Greenberg ME (1998)
$\mathrm{Ca}^{2+}$ influx regulates BDNF transcription by a CREB family transcription factor-dependent mechanism. Neuron 20:709-726. CrossRef Medline

Tian F, Marini AM, Lipsky RH (2010) NMDA receptor activation induces differential epigenetic modification of Bdnf promoters in hippocampal neurons. Amino Acids 38:1067-1074. CrossRef Medline

Vallejo M, Penchuk L, Habener JF (1992) Somatostatin gene upstream enhancer element activated by a protein complex consisting of CREB, Isl-1-like, and alpha-CBF-like transcription factors. J Biol Chem 267: 12876-12884. Medline

Wu X, Spiro C, Owen WG, McMurray CT (1998) cAMP response elementbinding protein monomers cooperatively assemble to form dimers on DNA. J Biol Chem 273:20820-20827. CrossRef Medline 